2020-05

\title{
Wave power extraction from a tubular structure integrated oscillating water column
}

\section{Zheng, Siming}

http://hdl.handle.net/10026.1/15279

10.1016/j.renene.2020.01.008

Renewable Energy

Elsevier

All content in PEARL is protected by copyright law. Author manuscripts are made available in accordance with publisher policies. Please cite only the published version using the details provided on the item record or document. In the absence of an open licence (e.g. Creative Commons), permissions for further reuse of content should be sought from the publisher or author. 


\title{
Wave power extraction from a tubular structure
} integrated oscillating water column

\author{
Siming Zheng a , Guixun $\mathrm{Zhu}^{\mathrm{a}}$, \\ David Simmonds ${ }^{\mathrm{a}}$, Deborah Greaves ${ }^{\mathrm{a}}$, Gregorio Iglesias ${ }^{\mathrm{a}, \mathrm{b}}$
}

Author names and affiliations:

Siming Zheng (corresponding author)

E-mail: siming.zheng@plymouth.ac.uk

Guixun Zhu

E-mail: guixun.zhu@plymouth.ac.uk

David Simmonds

E-mail: D.Simmonds@plymouth.ac.uk

Deborah Greaves

E-mail: deborah.greaves@plymouth.ac.uk

Gregorio Iglesias

E-mail: gregorio.iglesias@ucc.ie

a School of Engineering, Computing and Mathematics, University of Plymouth, Drake Circus, Plymouth PL4 8AA, United Kingdom

$b$ Centre for Marine and Renewable Energy Ireland (MaREI), Environmental Research Institute \& School of Engineering, University College Cork, Ireland

Received at Editorial Office: 18 Jan 2019

Article revised: 18 Nov 2019

Article accepted for publication: 2 Jan 2020

DOI: $10.1016 /$ j.renene.2020.01.008 


\begin{abstract}
Integrating wave energy converters with marine structures such as breakwaters, piles, and offshore wind turbines offers benefits in terms of wave power extraction, construction costs, and survivability. In this paper, the integration of an oscillating water column $(\mathrm{OWC})$ into a vertical tubular structure is considered. The OWC chamber is enclosed by the tubular-structure with its submerged side partially open to the sea. As ocean waves propagate through the device, an air turbine installed at the top of the chamber can be driven to extract wave power. An analytical model based on potential flow theory and the eigen-function matching method is developed to solve the wave scattering and radiation problems of the device in finite water depths. Wave excitation volume flux, hydrodynamic coefficients, optimal turbine damping and power capture factor are evaluated. Upon successful validation, the model is applied to investigate the effect of the radius and finite wall thickness of the tubular-structure, the size and position of the opening on wave power extraction. We find that a thinner chamber wall thickness offers benefits to wave power extraction in terms of a broader primary band of power capture factor response, and that a broader and higher capture factor band can be achieved by increasing the height of the vertical opening.
\end{abstract}

\title{
Keywords
}

Wave power; potential flow; analytical model; tubular structure; oscillating water column; hydrodynamics

\section{Introduction}

A large range of wave energy conversion concepts have been developed since 1970. These include: oscillating water column (OWC), point absorber, overtoping device, oscillating wave surge converter and attenuator device (Clément et al., 2002; Drew et al., 2009; Guo et al., 2018; López and Iglesias, 2014; Sheng et al., 2014a, b; Zheng and Zhang, 2017; Zheng and Zhang, 2018; Zheng et al., 2015). However, wave power exploitation is not as mature as the utilization of other renewable energies, such as solar power, wind power and tidal power. The high cost of construction and poor reliability could be two main challenges to put wave energy converters (WECs) into commercial application (Astariz and Iglesias, 2015; Mustapa et al., 2017).

At the present stage, integrating WECs into other existing marine structures is recognized to be a more realistic and reasonable approach than the deployment of isolated WECs (Contestabile et al., 2017; Ning et al., 2018; Perez-Collazo et al., 2018a, b; Viviano et al., 2016; Zhao et al., 2019). Compared to most other WECs, the only moving mechanical part of an OWC is a turbine located above the water, making it especially simple, reliable and easy to maintain. It is also relatively easy to integrate such devices into other oceanic structures (Falcão and Henriques, 2016; He et al., 2013; He and Huang, 2014; Henriques et al., 2013; López et al., 2016).

The integration of an OWC into a breakwater has been analytically investigated by many researchers. Evans and Porter (1995) studied the hydrodynamic characteristics of a two-dimensional (2D) OWC, which consisted of a thin vertical surface-piercing barrier in front of a vertical wall. Thanks to wave reflection from the wall, the results showed that, theoretically, all the incident wave power could be extracted by the OWC. Rezanejad et al. (2013) considered the effect of stepped bottom topography in improving the efficiency of a 2D nearshore thin-walled OWC device and 
revealed that a significantly increased capacity of power extraction might be achieved by the inclusion of an artificial step at the seabed along with some tuning. Later, Rezanejad et al. (2016) investigated the performance of a 2D dual-chamber nearshore OWC. The draft of the outside chamber was found to be important in determing the basic resonance frequency, which contributed significantly to the total power extraction. An analytical study of the 2D dual-chamber nearshore OWC placed over a stepped sea bed can be found in Rezanejad et al. (2015).

Regarding a tubular OWC, 3D theoretical models for solving hydrodynamic problems can be developed based on Bessel and modified Bessel functions in cylindrical coordinates (Zheng et al., 2019a; Zheng et al., 2019b). Wave power extraction by a thin-walled cylindrical OWC either installed at the tip of a long, thin breakwater or semi-embedded along a straight cliff-like coast was analytically studied by Martins-rivas and Mei (2009a, 2009b). The opening of the OWC side wall was extended from a specified submerged depth to the seabed. It was found that the power extracted by the OWC at the tip of a breakwater was insensitive to the incident wave direction, whereas the OWC along a straight wall was sensitive to it. These two analytical models were subsequently extended by Lovas et al. (2010) to examine a circular OWC installed at the tip of a coastal corner. Power extraction by the OWC at a convex and a concave corner was compared with those integrated into a thin breakwater and the straight coast examined before.

Apart from the integration of OWC into a breakwater, an OWC can be easily integrated into tubular oceanic structures, e.g., offshore wind turbine piles, SPAR platforms, piers and other piles. Deng et al. (2013) considered an OWC supported on a coaxial tubular structure with sector shaped opening extending from a given depth to the sea bed. An analytical model was developed to study the performance of this device using the assumption of thin walls. Model results showed that the device achieved its optimal performance in terms of power absorption when incident waves propagate perpendicular to the opening. A wave-flume study of a row of these devices and an analytical study of the effect of a V-shaped channel on improving power capture can be found in Xu and Huang (2018) and Deng et al. (2014), respectively.

In most of the analytical models above, which describethe integration of an OWC into either a breakwater or a tubular structure, the assumption of thin walls has been employed. This results in singular behaviour at the edges of the immersed openings. To deal with such singularities, the horizontal velocity across the gap was required to be expressed either as expansions of Chebyshev polynomials or cosine series. Ultimately, this required a large number of truncated terms in order to obtain accurate results. Additionaly, all OWCs were examined with openings which extended from a specified submerged depth down to the seabed. In the authors' opinion, the effect of the wall thickness of the OWC chamber is important and cannot be neglected in reality (Elhanafi et al., 2017; Morris-Thomas et al., 2007). Since most wave power (95\% approximately) is concentrated at no more than a fourth wavelength below the sea water level (Drew et al., 2009), in practice, it may not be essential to open the side wall of the OWC all the way to the seabed. What is more, either too large an opening size or too thin a chamber/tube structure wall works against the survivability of the OWC.

In this paper, the performance of an OWC integrated into a tubular structure is considered. We examine the performance of the OWC to variations in the geometry both of the OWC chamber and of the submerged OWC entrance. In particular, variations of height, angular width and depth of immersion of the entrance are considered. Power extraction of the device is investigated using an analytical model based on potential flow theory. An eigen-function matching method is developed 
which obviates the need restriction for the thin-walled assumption to be made, thus enabling the influence of the finite wall thickenss to be investigated. What is more, much fewer truncated eigenfunction expansion terms are required to obtain convergent results compared to the earlier model employing the thin-walled restriction. The new analytical model is first validated and then applied to examine the influence of geometry on the wave power absorption.

\section{Mathematical model}

The tubular structure with integrated OWC considered in this study is shown in Fig.1. The structure sits in water of constant depth $h$.The outer and inner radii of tubular cross section are denoted $R$ and $R_{\mathrm{i}}$, respectively. Thus the finite wall thickness of the OWC chamber is $R-R_{\mathrm{i}}$. The side wall of the tubular structure is partially open beneath submergence $d$ with an opening size $d_{0}$ vertically and an opening angle $\alpha=v \pi$ in the horizental plane. Subjected to incident waves, an oscillating water column is enclosed by the tubular structure. A linear Wells turbine is considered to be installed at the top of the device in order to extract wave power.

To describe the problem mathematically, a Cartesian coordinate system $O x y z$ is defined with the mean water surface being the $O x y$ plane, the $O z$-axis pointing vertically upward along the vertical axis of the tubular structure, and the $O x$-axis touching one side of the opening (see Fig. 1b). In addition, a cylindrical coordinate system $\operatorname{Or} \theta z$ is introduced with the $O z$-axis coinciding with that of the $O x y z$ coordinate system. As shown in Fig. 1, the opening region with a fan-shaped cross section can be defined in the $\operatorname{Or} \theta z$ system as: $r \in\left[R_{\mathrm{i}}, R\right], \theta \in[0, v \pi], \mathrm{z} \in\left[-h_{0},-d\right]$, in which $h_{0}=d+d_{0}$. The rest of the water domain can be divided into an inner region, i.e., $r \in\left[0, R_{\mathrm{i}}\right], \theta \in[0,2 \pi], \mathrm{z} \in[-$ $h, 0]$, and an outer region, i.e., $r \in[R, \infty), \theta \in[0,2 \pi], \mathrm{z} \in[-h, 0]$.

(a)

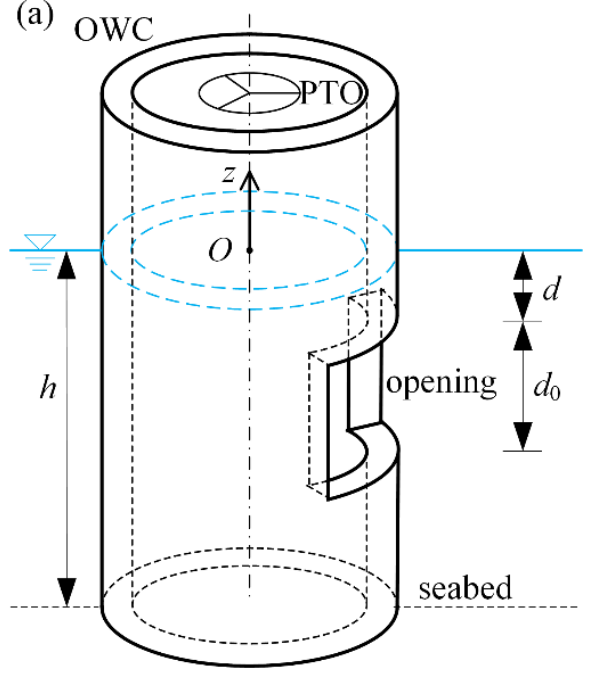

(b)

Fig. 1. Schematic diagram of a tubular structure integrated OWC: (a) side view; (b) top view.

When the device is subjected to monochromatic incident waves with small wave amplitude $A$, incident direction $\beta$ (see Fig. 1b) and angular wave frequency $\omega$, and assuming that water is inviscid and incompressible, then a velocity potential $\operatorname{Re}\left[\Phi(x, y, z) \mathrm{e}^{-\mathrm{i} \omega t}\right]$ might be used to describe the flow field. Here, i denotes the imaginary unit, $t$ represents time and $\Phi$ is the complex spatial velocity potential. Similarly, the air pressure inside the OWC chamber might be expressed as $\operatorname{Re}\left[p \mathrm{e}^{-\mathrm{i} \omega t}\right]$, where $p$ denotes the complex air pressure amplitude. $\Phi$ might be decomposed into the incident wave spatial potential, $\Phi_{\mathrm{I}}$, diffracted wave spatial potential, $\Phi_{\mathrm{D}}$, and the radiated wave spatial potential, 
$\Phi_{\mathrm{R}}:$

$$
\Phi=\Phi_{\mathrm{I}}+\Phi_{\mathrm{D}}+p \Phi_{\mathrm{R}}
$$

150

151

152

153

154

155

156

157

158

159

160

161

162

163

164

165

166

167

where $\Phi_{\mathrm{R}}$ represents the radiated spatial potential induced by unit air pressure oscillation inside the OWC chamber. Both $\Phi_{\mathrm{D}}$ and $\Phi_{\mathrm{R}}$ satisfy the Laplace equation in the water domain and a radiation condition at infinite distance. $\Phi_{\mathrm{I}}$ is generally well known and can be expressed in the cylindrical coordinate system $\operatorname{Or} \theta z$ as

$$
\Phi_{\mathrm{I}}(r, \theta, z)=-\frac{\mathrm{i} g A}{\omega} \frac{Z_{0}(z)}{Z_{0}(0)} \sum_{m=-\infty}^{\infty} \mathrm{i}^{m} \mathrm{e}^{-\mathrm{i} m \beta} J_{m}\left(k_{0} r\right) \mathrm{e}^{\mathrm{i} m \theta},
$$

in which $g$ is the gravity acceleration, $k_{0}$ is the wave number, $J_{m}$ is the Bessel function and

$$
Z_{0}(z)=\cosh \left[k_{0}(z+h)\right]\left\{\frac{1}{2}\left[1+\frac{\sinh \left(2 k_{0} h\right)}{2 k_{0} h}\right]\right\}^{-0.5} .
$$

For the sake of simplicity, hereinafter, the scattering velocity potential $\Phi_{\mathrm{S}}$ is adopted to represent the sum of the incident and diffracted velocity potentials, i.e., $\Phi_{\mathrm{S}}=\Phi_{\mathrm{I}}+\Phi_{\mathrm{D}}$.

\subsection{Boundary conditions}

The boundary conditions that $\Phi \chi(\chi=\mathrm{S}, \mathrm{R})$ should satisfy are as follows:

$$
\frac{\partial \Phi_{\chi}}{\partial z}=0, \quad z=-h
$$

$$
\frac{\partial \Phi_{\chi}}{\partial z}=0, \quad r \in[R \mathrm{i}, R], \theta \in[0, v \pi], z=-h_{0} \text { and }-d,
$$

$$
\frac{\partial \Phi_{\chi}}{\partial r}=0, \quad r=R_{\mathrm{i}} \text { and } R, \theta \notin[0, v \pi], z \in\left[-h_{0},-d\right],
$$

$$
\frac{\partial \Phi_{\chi}}{\partial r}=0, \quad r=R_{\mathrm{i}} \text { and } R, \theta \in[0,2 \pi], z \in\left[-h,-h_{0}\right] \cup[-d, 0] \text {, }
$$

where $\rho$ is the water density.

\subsection{Expressions of $\Phi_{\chi}$ in different regions}




$$
\tilde{K}_{m}\left(k_{l} r\right)=\left\{\begin{array}{ll}
H_{m}\left(k_{l} r\right), & l=0 \\
K_{m}\left(k_{l} r\right), & l \neq 0
\end{array},\right.
$$

where $A_{m, l}^{\chi}$ is the unknown coefficients to be determined; $\Phi_{\mathrm{p}, \chi}^{\mathrm{in}}$ is a particular solution, $\Phi_{\mathrm{p}, \mathrm{S}}^{\mathrm{in}}=0$, whereas $\Phi_{\mathrm{p}, \mathrm{R}}^{\mathrm{in}}=-\mathrm{i} /(\rho \omega)$;

$$
\tilde{I}_{m}\left(k_{l} r\right)= \begin{cases}J_{m}\left(k_{l} r\right), & l=0 \\ I_{m}\left(k_{l} r\right), & l \neq 0\end{cases}
$$

in which $I_{\mathrm{m}}$ denotes the modified Bessel function of the first kind; $k_{l}$ and $Z_{l}$ are the eigenvalue and eigen-function $(l>0)$ given by

$$
\omega^{2}=-k_{l} g \tan \left(k_{l} h\right), \quad l=1,2,3, \ldots
$$

$$
Z_{l}(z)=\cos \left[k_{l}(z+h)\right]\left\{\frac{1}{2}\left[1+\frac{\sin \left(2 k_{l} h\right)}{2 k_{l} h}\right]\right\}^{-0.5}
$$

II, opening region with a fan-shaped cross section

$$
\Phi_{\chi}^{\text {open }}(r, \theta, z)=\sum_{m=0}^{\infty}\left[F_{m, 0}^{\chi}(r)+\sum_{l=1}^{\infty}\left(C_{m, l}^{\chi} \frac{\frac{I_{m}}{v}\left(\beta_{l} r\right)}{I_{\frac{m}{v}}\left(\beta_{l} R\right)}+D_{m, l}^{\chi} \frac{K_{\frac{m}{v}}\left(\beta_{l} r\right)}{K_{\frac{m}{v}}\left(\beta_{l} R\right)}\right) \cos \left[\beta_{l}\left(z+h_{0}\right)\right]\right] \cos \left(\frac{m \theta}{v}\right),
$$

where

$C_{m, l}^{\chi}$ and $D_{m, l}^{\chi}$ are the coefficients to be solved; $K_{m}$ is the modified Bessel function of the second kind; $\quad \beta_{l}$ is the $l$-th eigenvalue given by

$$
\beta_{l}=\frac{l \pi}{h_{0}-d}, l=0,1,2,3, \ldots
$$

III, outside region

$$
\Phi_{\chi}^{\text {out }}(r, \theta, z)=\sum_{m=-\infty}^{\infty} \sum_{l=0}^{\infty} E_{m, l}^{\chi} \frac{\tilde{K}_{m}\left(k_{l} r\right)}{\tilde{K}_{m}\left(k_{l} R\right)} Z_{l}(z) \mathrm{e}^{\mathrm{i} m \theta}+\Phi_{\mathrm{p}, \chi}^{\text {out }},
$$

in which $E_{m, l}^{\chi}$ is the unknown coefficients to be determined; and $\Phi_{\mathrm{p}, \chi}^{\text {out }}$ is a particular solution, $\Phi_{\mathrm{p}, \mathrm{S}}^{\text {out }}=\Phi_{\mathrm{I}}$, whereas $\Phi_{\mathrm{p}, \mathrm{R}}^{\text {out }}=0$;

where $H_{m}$ denotes the Hankel function of the first kind. 


\subsection{Method of computation for unknown coefficients}

The spatial potentials in different regions as given in Section 2.2 satisfy Eqs. (4) (8). The other boundary conditions, i.e., Eqs. (9) (10), together with the velocity and pressure continuity conditions on the interfaces of adjacent regions should all be satisfied, which can be used to calculate the unknown coefficients in $\Phi \chi$. Specifically, these equations are as follows:

1) Continuity of normal velocity at the boundary $r=R_{\mathrm{i}}$ :

$$
\frac{\partial \Phi_{\chi}^{\text {in }}}{\partial r}=\left\{\begin{array}{lr}
0, & r=R_{\mathrm{i}}, \theta \in[0,2 \pi], z \in[-d, 0] \cup\left[-h,-h_{0}\right] ; \\
\text { and } r=R_{\mathrm{i}}, \theta \in[v \pi, 2 \pi], z \in\left[-h_{0},-d\right] & \\
\frac{\partial \Phi_{\chi}^{\text {open }}}{\partial r}, & r=R_{\mathrm{i}}, \theta \in[0, v \pi], z \in\left[-h_{0},-d\right]
\end{array} .\right.
$$

2) Continuity of normal velocity at the boundary $r=R$ :

$$
\frac{\partial \Phi_{\chi}^{\text {out }}}{\partial r}=\left\{\begin{array}{lr}
0, & r=R, \theta \in[0,2 \pi], z \in[-d, 0] \cup\left[-h,-h_{0}\right] ; \\
& \text { and } r=R, \theta \in[v \pi, 2 \pi], z \in\left[-h_{0},-d\right] . \\
\frac{\partial \Phi_{\chi}^{\text {open }}}{\partial r}, & r=R, \theta \in[0, v \pi], z \in\left[-h_{0},-d\right]
\end{array} .\right.
$$

3) Continuity of pressure at the boundary $r=R_{\mathrm{i}}$ :

$$
\Phi_{\chi}^{\mathrm{open}}=\Phi_{\chi}^{\mathrm{in}}, \quad r=R_{\mathrm{i}}, \theta \in[0, v \pi], z \in\left[-h_{0},-d\right] .
$$

4) Continuity of pressure at the boundary $r=R$ :

$$
\Phi_{\chi}^{\text {out }}=\Phi_{\chi}^{\text {open }}, \quad r=R, \theta \in[0, v \pi], z \in\left[-h_{0},-d\right] .
$$

After inserting expressions of $\Phi \chi$ as given in Section 2.2, i.e., Eqs.(11), (15) and (18), into Eqs. (20) (23), and making use of orthogonality of eigen-functions and trigonometric functions, the unknown coefficients in $\Phi \chi$ can be determined. Details of the derivation can be found in Appendix A.

\subsection{Excitation volume flow}

Once the unknown coefficients are determined, the excitation volume flow $Q_{\mathrm{e}}$, which is the rate of upward displacement of the water surface inside the column contributed by the scattering potential, can be calculated in terms of $A_{m, l}^{\mathrm{S}}$ by:

$$
Q_{\mathrm{e}}=\left.\int_{0}^{2 \pi} \int_{0}^{R_{\mathrm{i}}} \frac{\partial \Phi_{\mathrm{S}}}{\partial z}\right|_{z=0} r \mathrm{~d} r \mathrm{~d} \theta=\frac{2 \pi \omega^{2} R_{\mathrm{i}}}{g}\left(-\frac{A_{0,0}^{\mathrm{S}}}{k_{0}^{2}} Z_{0}(0)+\sum_{l=1}^{\infty} \frac{A_{0, l}^{\mathrm{S}}}{k_{l}^{2}} Z_{l}(0)\right) .
$$

\subsection{Hydrodynamic coefficients}

In a similar way, the hydrodynamic coefficients $c$ and $a$, which are also known as the radiation conductance and the radiation susceptance (Falnes, 2002), can be derived from the volume flow inside the OWC chamber induced by the radiated potential, $Q_{\mathrm{R}}$, in terms of $A_{m, l}^{\mathrm{R}}$ as: 


$$
-(c-\mathrm{i} a)=Q_{\mathrm{R}}=\left.\int_{0}^{2 \pi} \int_{0}^{R_{\mathrm{i}}} \frac{\partial \Phi_{\mathrm{R}}}{\partial z}\right|_{z=0} r \mathrm{~d} r \mathrm{~d} \theta=\frac{2 \pi \omega^{2} R_{\mathrm{i}}}{g}\left(-\frac{A_{0,0}^{\mathrm{R}}}{k_{0}^{2}} Z_{0}(0)+\sum_{l=1}^{\infty} \frac{A_{0, l}^{\mathrm{R}}}{k_{l}^{2}} Z_{l}(0)\right) .
$$

\subsection{Wave power extraction}

A linear Wells turbine is considered as the power take-off (PTO) system. Hence the mass flux through the turbine might be assumed proportional to the chamber air pressure following Martinsrivas and Mei (2009a); Sarmento and Falcão (1985). Therefore, the complex air pressure amplitude $p$ can be related to the scattering and radiation problems by:

$$
\left[-\mathrm{i}\left(a+a_{\text {РTO }}\right)+\left(c+c_{\text {РTO }}\right)\right] p=Q_{\mathrm{e}},
$$

where $a_{\mathrm{PTO}}=\omega V_{0} /\left(v^{2} \rho_{0}\right)$ is a parameter used for taking into account air compressibility, in which $V_{0}$ represents chamber volume, $v$ is the sound velocity in air and $\rho_{0}$ denotes the static air density; $c_{\text {PTO }}$ is the damping coefficient induced by the Wells turbine, which is sensitive to the rotational speed of turbine blades, the scales and setup of the turbine, as well as air density. Following Lovas et al. (2010); Martins-rivas and Mei (2009a), $\rho / \rho_{0}=1000, v=340 \mathrm{~m} / \mathrm{s}, g=9.81 \mathrm{~m} / \mathrm{s}^{2}, h=10 \mathrm{~m}$ and $V_{0}=$ $\pi R^{2} h$ are adopted in this paper.

The time-averaged pneumatic power can be evaluated by:

$$
P=\frac{c_{\text {PTO }}}{2}|p|^{2}=\frac{c_{\text {PTO }}}{2} \frac{\left|Q_{\mathrm{e}}\right|^{2}}{\left(a+a_{\mathrm{PTO}}\right)^{2}+\left(c+c_{\mathrm{PTO}}\right)^{2}},
$$

in which the PTO damping is selected for maximizing power absorption, i.e., by satisfying the so called "optimum amplitude condition", $\partial P / \partial c_{\mathrm{PTO}}=0$ (Falnes, 2002). The optimal $c_{\text {PTO }}$ is given by

$$
c_{\mathrm{PTO}}=\sqrt{\left(a+a_{\mathrm{PTO}}\right)^{2}+c^{2}} \text {. }
$$

More often, wave power extraction of the $\mathrm{OWC}$ is further evaluated in terms of the nondimensional parameter, wave power capture factor $\eta$, as

$$
\eta=k L=\frac{k P}{P_{\text {in }}}=\frac{2 k P}{\rho g A^{2} c_{g}}
$$

where $L$ is the so-called capture length, $P_{\text {in }}$ represents incident wave power per unit width of the water front; $c_{g}$ is the wave group velocity and, in subsequent computations, $k$ is used to represent $k_{0}$ for convenience.

\section{Validation}

Following Lovas et al. (2010), hereinafter, $Q_{\mathrm{e}}, c, a, c_{\mathrm{PTO}}$ and $a_{\mathrm{PTO}}$ are normalized as follows:

$$
\bar{Q}_{\mathrm{e}}=\frac{\sqrt{g / h}}{A h g} Q_{\mathrm{e}} ; \quad\left(\bar{c}, \bar{a}, \bar{c}_{\mathrm{PTO}}, \bar{a}_{\mathrm{PTO}}\right)=\frac{\rho \sqrt{g / h}}{h}\left(c, a, c_{\mathrm{PTO}}, a_{\mathrm{PTO}}\right) .
$$

When $\alpha=2 \pi$ is employed with $h_{0}=h$, i.e., $d+d_{0}=h$, the device subjected to incident waves with either $\beta=0$ or $\pi$, works the same as a traditional fixed isolated OWC consisting of a truncated hollow cylinder, the hydrodynamic problem of which has already been numerically studied by Nader (2013) based on a Finite Element Method (FEM). Whereas when $R_{\mathrm{i}} \rightarrow R$ and $h_{0}=h$, i.e., $d+d_{0}=h$, the device turns into the object investigated by Deng et al. (2013). These two special cases can be used to validate the present analytical model. Figs. 2 and 3 give comparisons of the present analytical results 
of two selected cases, i.e., case I: $R / h=0.25,\left(R-R_{\mathrm{i}}\right) / h=0.05, \alpha=2.0 \pi, d_{0} / h=0.8, d / h=0.2$; case II: $R / h=0.5, \alpha=0.75 \pi, d_{0} / h=0.8, d / h=0.2, \beta=(1+0.5 v) \pi$, with those of Nader $(2013)$ and Deng et al. (2013), respectively.

(a)

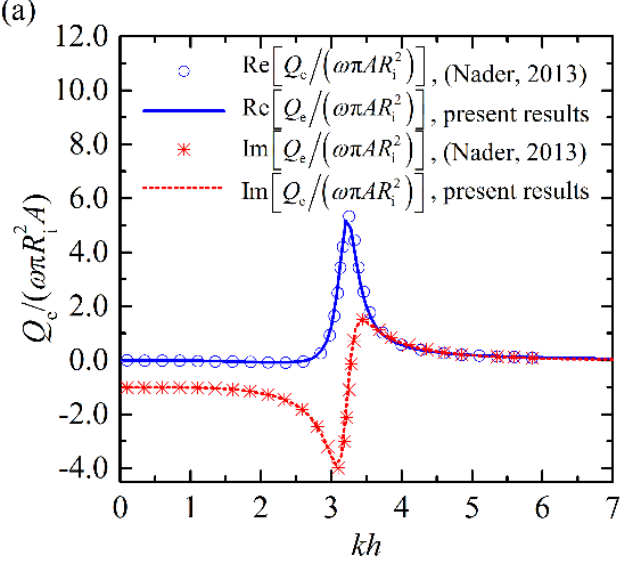

(b)

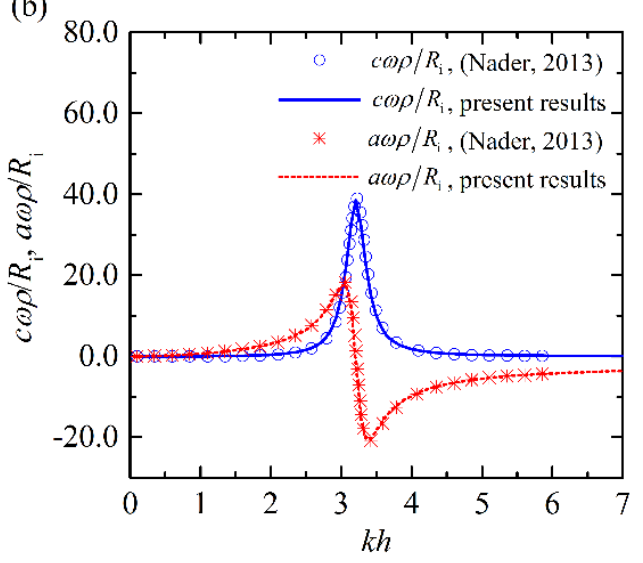

Fig. 2. Comparison of wave excitation volume flux and hydrodynamic coefficients with the numerical results (Nader, 2013) for $R / h=0.25,\left(R-R_{\mathrm{i}}\right) / h=0.05, \alpha=2.0 \pi, d_{0} / h=0.8, d / h=0.2$. (a) $Q_{\mathrm{e}}$; (b) $c$ and $a$.

(a)

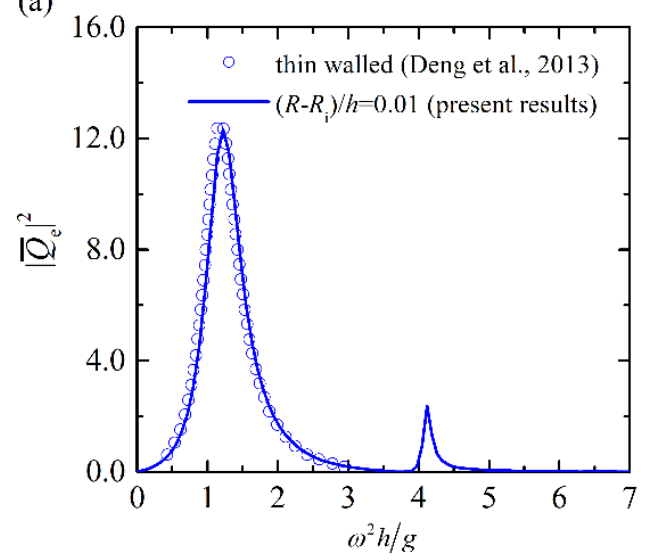

(c)

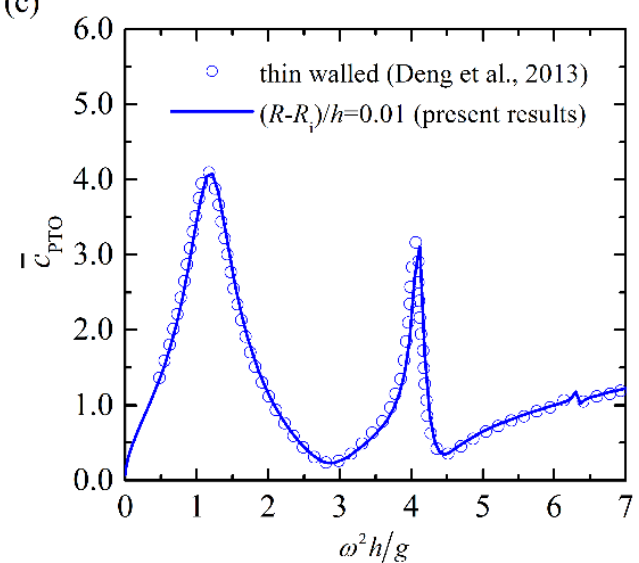

(b)

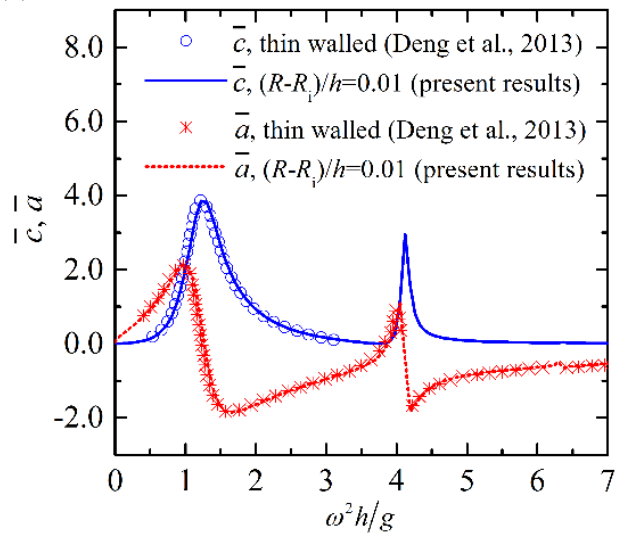

(d)

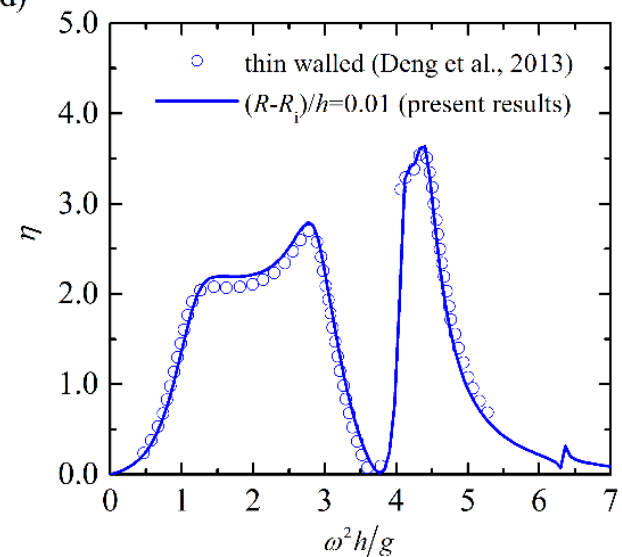

Fig. 3. Comparison of wave excitation volume flux, hydrodynamic coefficients, optimized PTO damping and power capture factor with the previous analytical results based on thin walled 
assumption (Deng et al., 2013) for $R / h=0.5, \alpha=0.75 \pi, d_{0} / h=0.8, d / h=0.2, \beta=(1+0.5 v) \pi$. (a) $\left|\bar{Q}_{\mathrm{e}}\right|^{2}$; (b) $\bar{c}$ and $\bar{a}$; (c) $\bar{c}_{\mathrm{PTO}} ;$ (d) $\eta$.

In addition to the comparison of the present results with published numerical/analytical data (Deng et al., 2013; Nader, 2013), the present analytical model is also validated against the experimental data (Bosma et al., 2017) of a traditional fixed isolated OWC with no top on the device, $R_{\mathrm{i}}=0.31 \mathrm{~m}, R=0.32 \mathrm{~m}, d=0.443 \mathrm{~m}, h=1.36 \mathrm{~m}, h_{0}=h, \alpha=2 \pi$, and $\beta=0$. The frequency response of the average wave amplitude inside the OWC in terms of $\left|Q_{\mathrm{e}}\right| /\left(\omega \pi R_{\mathrm{i}}{ }^{2} A\right)$ is illustrated in Fig. 4. The excellent agreement (Figs. 2, 3, and4) proves that the present analytical model works well in solving the scattering/radiation problems and evaluating power absorption.

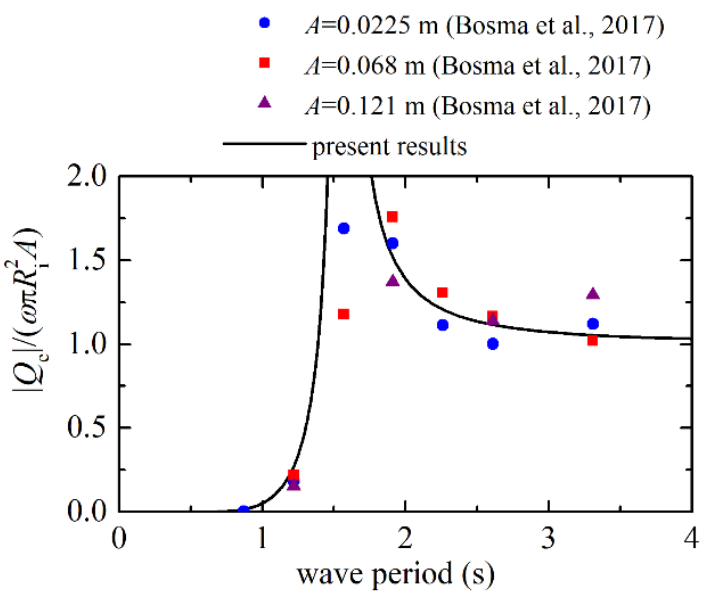

Fig. 4. The averaged wave amplitude inside the OWC in terms of $\left|Q_{\mathrm{e}}\right| /\left(\omega \pi R_{\mathrm{i}}^{2} A\right)$ compared with experimental data (Bosma et al., 2017) for $R_{\mathrm{i}}=0.31 \mathrm{~m}, R=0.32 \mathrm{~m}, d=0.443$ $\mathrm{m}, h=1.36 \mathrm{~m}, h_{0}=h, \alpha=2 \pi$, and $\beta=0$.

\section{Results and discussion}

In this section, the effects of the geometry of the tubular structure integrated OWC on $\left|\bar{Q}_{\mathrm{e}}\right|$, $\bar{c}, \bar{a}, \bar{c}_{\mathrm{PTO}}$ and $\eta$ are investigated with the validated analytical model. Since the optimal power absorption occurs when incident waves propagate perpendicular to the opening (Deng et al., 2013), $\beta=(1+0.5 v) \pi$ is employed in all the cases examined in the present section.

\subsection{Radius of the chamber, $R$}

For five different radii of the chamber with $R / h$ values $0.3,0.4,0.5,0.6$ and 0.7 , and $(R$ $\left.R_{\mathrm{i}}\right) / h=0.1, \alpha=1.0 \pi, d_{0} / h=0.3, d / h=0.2$, Fig. 5 presents how $\left|\bar{Q}_{\mathrm{e}}\right|, \bar{c}, \bar{a}, \bar{c}_{\text {РTO }}$ and $\eta$ vary with $k h$.

For relatively small columns, e.g., $R / h=0.3$, the $\left|\bar{Q}_{\mathrm{e}}\right|$ curve has only one single peak in the computed range of $k h$ (see Fig. 5a). For larger radii, more peaks can be observed, e.g., for $R / h=0.4$, there are two peaks, and for $R / h=0.7$, there are three. These peaks might be identified with the natural modes inside a closed cylinder. These can be computed using the method outlined in Lovas et al. (2010). As $R / h$ increases, these peaks, especially the main ones, are higher and the corresponding $k h$ are smaller. This is reasonable when considering the depth normalized horizontal scale of the tubular cross section $(R / h)$ in comparison to the wavelength. 
Variations of hydrodynamic coefficients $\bar{c}$ and $\bar{a}$ with $k h$ are plotted in Figs. 5b and 5c. Similar to $\left|\bar{Q}_{\mathrm{e}}\right|, \bar{c}$ curve for any specified $R / h$ is a single-peak or multi-peak curve; whereas the $\bar{a}$ curve is shaped like one or more letters "N". For the $\bar{c}$ curve with two or more peaks, $\bar{c}$ vanishes at certain $k h$ between those relating to every two adjacent peaks. The $k h$ where $\bar{c}$ peaks and $\bar{a}$ changes sign not only coincide with each other, but also agree with that where the peak of $\left|\bar{Q}_{\mathrm{e}}\right|$ occurs. In Fig. 5c, each of the thin solid lines denotes the $-\bar{a}_{\mathrm{PTO}}-k h$ curve that corresponds to the line of $\bar{a}$ plotted in the same color for the same device. The intersection points of $\bar{a}$ and $-\bar{a}_{\text {РTO }}$ curves refer to the "optimum phase condition" or the "resonance condition", for which it can be seen from Eqs. (26) (27) that $p$ is in phase with $Q_{\mathrm{e}}$, and the maximum of power absorption is obtained. As $R / h$ increases, the first two intersection points between the $\bar{a}$ and $-\bar{a}_{\text {PTO }}$ curves shift to lower frequency and are closer together. With the increase of $R / h$, the corresponding primary band of the $\eta$ curve also moves towards lower frequency and gets narrower. The $\bar{c}_{\text {PTO }}$ curves as given in Fig. $5 \mathrm{~d}$ show that the larger the value of $R / h$, the higher and more abrupt the variation of $\bar{c}_{\text {PTO }}$ for $k h$ in the range of $(0,3.0]$, leading to more demanding in the design of the PTO control system.

(a)

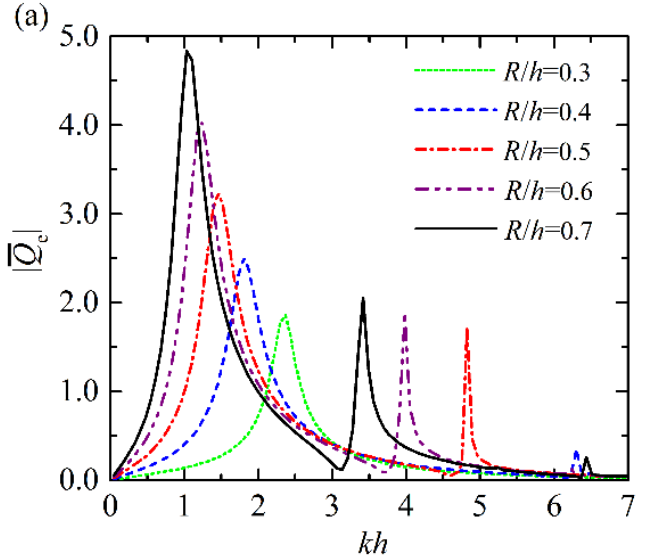

(c)

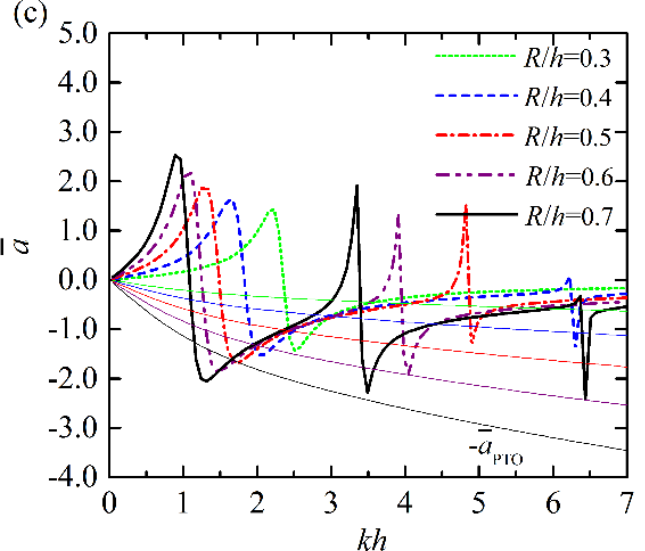

(b)

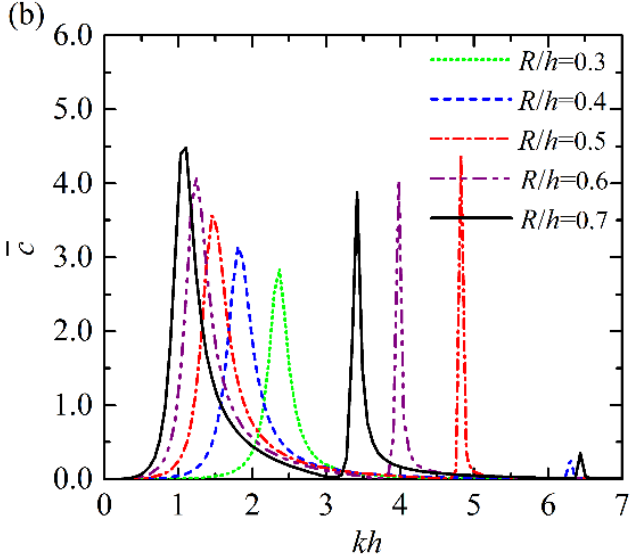

(d)

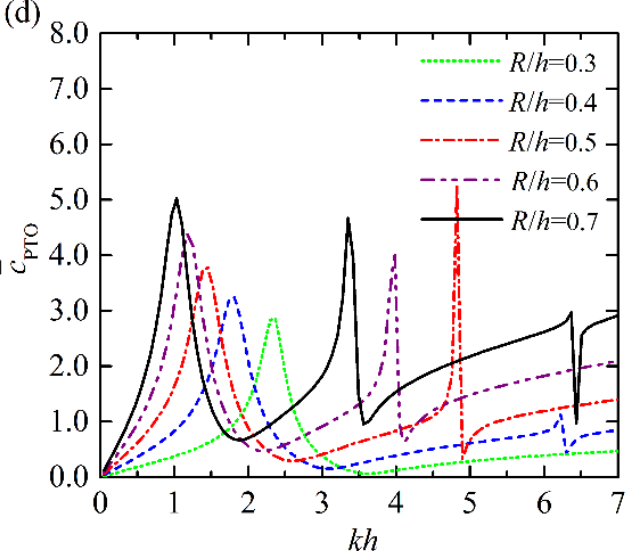




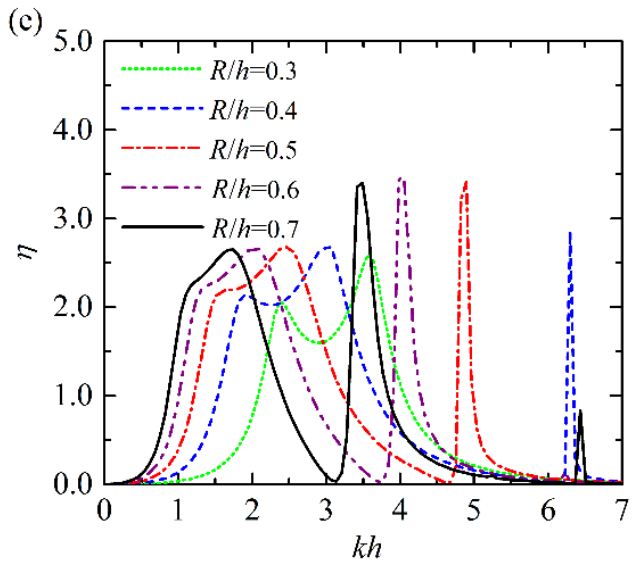

Fig. 5. Comparison for different radii of the OWC chamber, $R / h=0.3,0.4,0.5,0.6,0.7$. (a) $\left|\bar{Q}_{\mathrm{e}}\right|$; (b) $\bar{c}$; (c) $\bar{a}$ and $-\bar{a}_{\mathrm{PTO}}$ (thin solid lines, each of which corresponds to the line of $\bar{a}$ plotted in the same color); (d) $\bar{c}_{\text {PTO }}$; (e) $\eta$. In every case, $\left(R-R_{\mathrm{i}}\right) / h=0.1, \alpha=1.0 \pi, d_{0} / h=0.3, d / h=0.2$.

4.2 Wall thickness of the chamber, $R-R_{\mathrm{i}}$

Fig. 6 presents how $\left|\bar{Q}_{\mathrm{e}}\right|, \bar{c}, \bar{a}, \bar{c}_{\text {РTO }}$ and $\eta$ vary with $k h$ for four different wall thicknesses of the chamber with $\left(R-R_{\mathrm{i}}\right) / h$ values $0.05,0.1,0.15$ and 0.2 , and $R / h=0.5, \alpha=1.0 \pi, d_{0} / h=0.3, d / h=0.2$. Since the outer radius $R$ is fixed, variation of the thickness works by changing the value of $R_{\mathrm{i}}$.

For each of the four cases tested, the $\left|\bar{Q}_{\mathrm{e}}\right|$ curve has two peaks (see Fig. 6a). As $\left(R-R_{\mathrm{i}}\right) / h$ increases from 0.05 to 0.2 , the first peak is lower and narrower, and moves towards higher frequency. The $k h$ where the second peak occurs also increases with the increase of $\left(R-R_{\mathrm{i}}\right) / h$, and even more dramatically compared to that of the first peak. Similar features are found for $\bar{c}$ as well, as illustrated in Fig. 6b. In Fig. 6c, for the parameter accounting for air compressibility, $a_{\text {PTO, depends }}$ critically on chamber volume $V_{0}$, which is fixed in a given design (i.e., $V_{0}=\pi R^{2} h ; R / h=0.5$ ) regardless of the value of $\left(R-R_{\mathrm{i}}\right) / h$, the $-\bar{a}_{\text {PTO }}$ curves in subsequent computations, are all the same and overlap each other. Clearly larger $\left(R-R_{\mathrm{i}}\right) / h$ leads to narrower spacing between the first two intersection points of the $\bar{a}$ and $-\bar{a}_{\text {PTO }}$ curves. For larger values of $\left(R-R_{\mathrm{i}}\right) / h$, although the first peak value of $\bar{c}_{\text {РTO }}$ as shown in Fig. 6d is smaller, the variation of $\bar{c}_{\text {PTO }}$ is found more sensitive to $k h$ for $k h \in[1.5,3.0]$, which might increase requirements for the PTO control system. As illustrated in Fig. 6e, the device with a smaller wall thickness of OWC chamber offers obvious benefits to wave power extraction in terms of a wider primary band of $\eta$ curves without changing frequency position of the band significantly. What is more, the second band of $\eta$ curves is broadened as well, while the corresponding $k h$ turns smaller. Although the wall thickness of the OWC chamber should be as small as possible to achieve a broad-bandwidth power absorption response, in practice, the wall of the OWC chamber cannot be too thin, otherwise the structural strength and device survivability are threatened. 

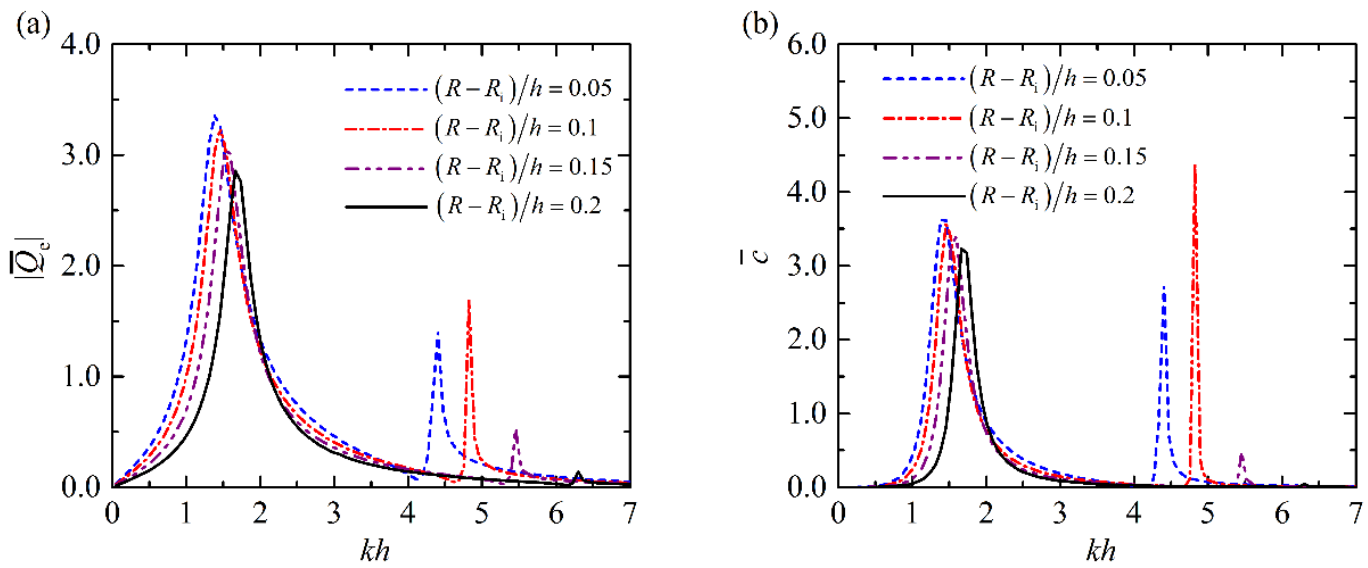

341
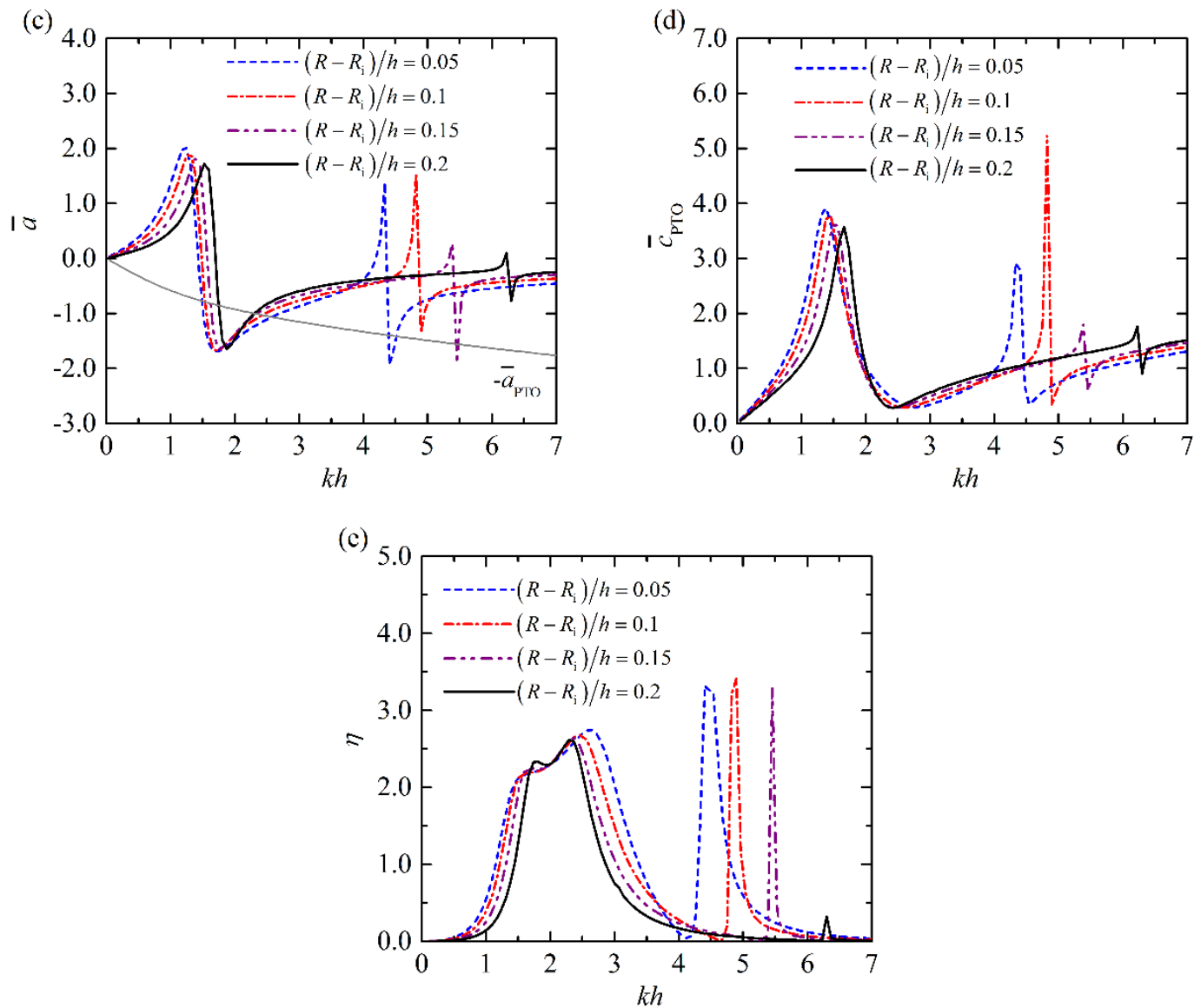

Fig. 6. Comparison for different wall thickness of the chamber, $\left(R-R_{\mathrm{i}}\right) / h=0.05,0.1,0.15,0.2$. (a) $\left|\bar{Q}_{\mathrm{e}}\right| ;$ (b) $\bar{c}$; (c) $\bar{a}$ and $-\bar{a}_{\mathrm{PTO}}$ (gray solid line); (d) $\bar{c}_{\mathrm{PTO}}$; (e) $\eta$. In every case, $R / h=0.5$, $\alpha=1.0 \pi, d_{0} / h=0.3, d / h=0.2$.

\subsection{Opening size in terms of $\alpha$}

The effect of the opening size in terms of $\alpha$ on $\left|\bar{Q}_{\mathrm{e}}\right|, \bar{c}, \bar{a}, \bar{c}_{\mathrm{PTO}}$ and $\eta$ are illustrated in Fig. 7 for $R / h=0.5,\left(R-R_{\mathrm{i}}\right) / h=0.1, d_{0} / h=0.3, d / h=0.2$. Five cases with $\alpha=0.5 \pi, 0.75 \pi, 1.0 \pi, 1.25 \pi$ and $1.5 \pi$ are examined.

As shown in Fig.7a, increasing the opening size in terms of $\alpha$ results in a larger $k h$ where the main peak of $\left|\bar{Q}_{\mathrm{e}}\right|$ occurs, and leads to the main peak being lower although broader. As a comparison, as plotted in Fig. 7b, the main peak of $\bar{c}$ is generally higher with $\alpha$ increasing from 
$0.75 \pi$ to $1.5 \pi$, which is reasonable as the radiation loss becomes greater. As shown in Fig. 7c, the first two resonance frequencies, together with their difference, increase dramatically with the increase of $\alpha$. It is seen from Figs. 7a 7c that the $k h$ corresponding to the second peaks of $\left|\bar{Q}_{\mathrm{e}}\right|$ and $\bar{c}$, and also regarding to the second sign changing point of $\bar{a}$, is almost independent of $\alpha$.

Variation of $\bar{c}_{\text {PTO }}$ and the corresponding $\eta$ are presented in Figs. 7d and 7e. As $\alpha$ increases from $0.5 \pi$ to $1.5 \pi$, the primary band of the $\eta$ curve shifts towards higher frequency. Although the main resonance peak of $\eta$ can be basically widened from the view of the difference between the first two resonance frequencies, meanwhile the peak value of $\eta$ and the strength of the tubular structure can be weakened. An appropriate value of $\alpha$ should be selected neither too small to capture wave power, nor too large to ensure structural strength. The results given in Fig. 7e shows that the largest value of $\eta$ and the corresponding $k h$ for $\alpha=0.5 \pi, 0.75 \pi, 1.0 \pi, 1.25 \pi$ and $1.5 \pi$ are $(2.07,1.52),(2.39$, $1.95),(2.68,2.44),(2.49,2.86)$, and $(1.87,2.09)$, respectively.

(a)

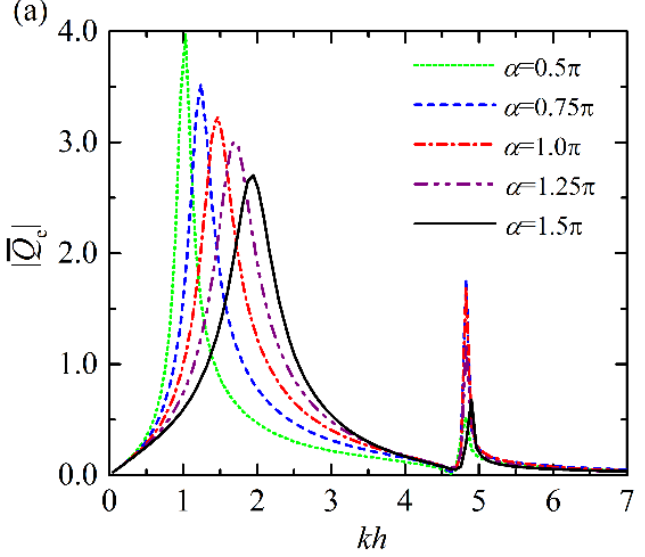

(c)

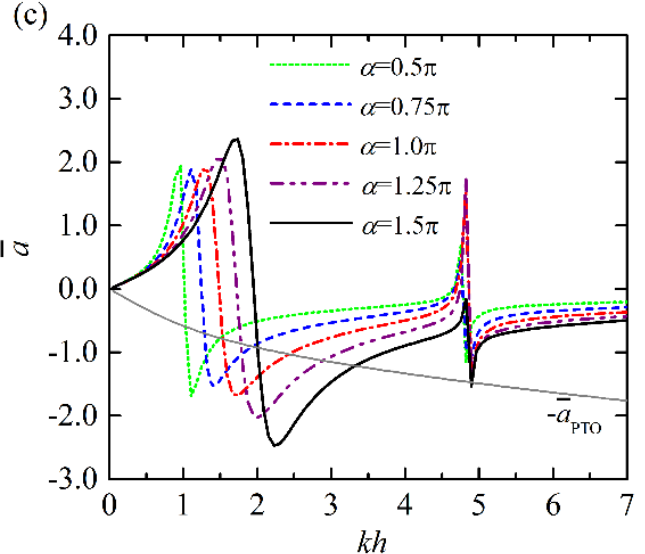

(b)

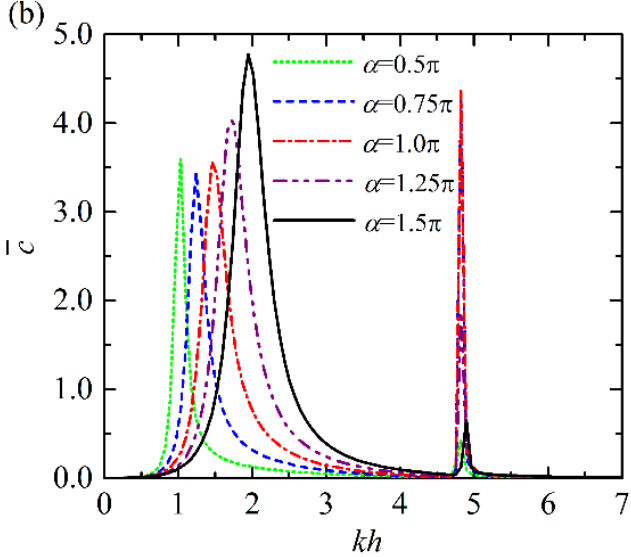

(d)

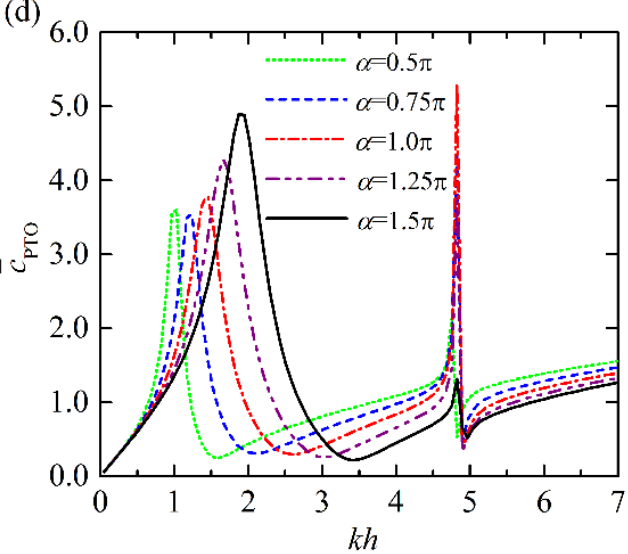




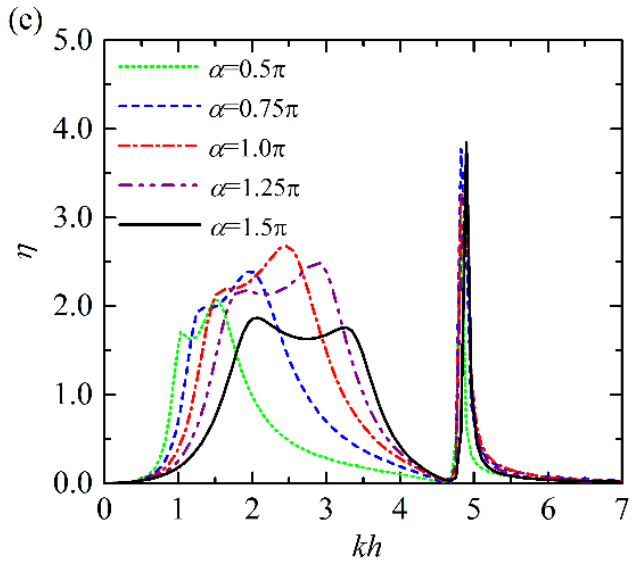

Fig. 7. Comparison for different opening size of the OWC chamber in terms of $\alpha=0.5 \pi, 0.75 \pi$, $1.0 \pi, 1.25 \pi, 1.5 \pi$. (a) $\left|\bar{Q}_{\mathrm{e}}\right|$; (b) $\bar{c}$; (c) $\bar{a}$ and $-\bar{a}_{\mathrm{PTO}}$ (gray solid line); (d) $\bar{c}_{\mathrm{PTO}}$; (e) $\eta$. In every case, $R / h=0.5,\left(R-R_{\mathrm{i}}\right) / h=0.1, d_{0} / h=0.3, d / h=0.2$.

The free surface motion near the tubular structure integrated OWC due to different value of $\alpha$ can be calculated by means of the analytical model, as $\xi_{\text {time }}(r, \theta, t)=\operatorname{Re}\left[\xi(r, \theta) \mathrm{e}^{-\mathrm{i} \omega t}\right]$, in which $\xi(r, \theta)=(\mathrm{i} \omega / g) \Phi(r, \theta, 0)$ outside the OWC chamber and $\xi(r, \theta)=(\mathrm{i} \omega / g)[\Phi(r, \theta, 0)+\mathrm{i} p /(\rho \omega)]$ inside the chamber. Particularly, for $\alpha=0$, the tubular structure integrated OWC works like a traditional bottom mounted solid cylinder and the corresponding $\xi(r, \theta)$ in the outside region is well known as:

$$
\xi(r, \theta)=A \sum_{m=-\infty}^{\infty} \mathrm{i}^{m} \mathrm{e}^{-\mathrm{i} m \pi}\left(J_{m}(k r)-\frac{J_{m}^{\prime}(k R)}{H_{m}^{\prime}(k R)} H_{m}(k r)\right) \mathrm{e}^{\mathrm{i} m \theta}
$$

Let us take $k h=2.5$ as an example. Fig. 8 illustrates the contour plot of normalized wave amplitude inside and outside of the tubular structure integrated OWC with $\alpha=0,0.5 \pi, 0.75 \pi, 1.0 \pi$, $1.25 \pi$ and $1.5 \pi$, and $R / h=0.5,\left(R-R_{\mathrm{i}}\right) / h=0.1, d_{0} / h=0.3, d / h=0.2$, for such wave condition (i.e., $k h=2.5$ ). For the sake of comparison, each subfigure in Fig. 8 has been rotated clockwise by an angle of $0.5 \alpha$, hence the symmetrical lines of the opening in these OWCs coincide with each other and meanwhile all the OWCs are subjected to the incident waves propagating in the same direction, i.e., from right to left. As expected, when the tubular structure is side open, the water column enclosed starts oscillating. The wave amplitude at the innermost region of the OWC chamber $\left(r=R_{\mathrm{i}}, \theta=\pi\right)$ is maximum inside the chamber due to full wave reflection, whereas the minimum is observed at the opening ( $r=R_{\mathrm{i}}, \theta=0$ ). The larger the opening size, the stronger the wave motion within the chamber. The largest wave motion outside the OWC occurs in front of the opening regardless of the opening size. 

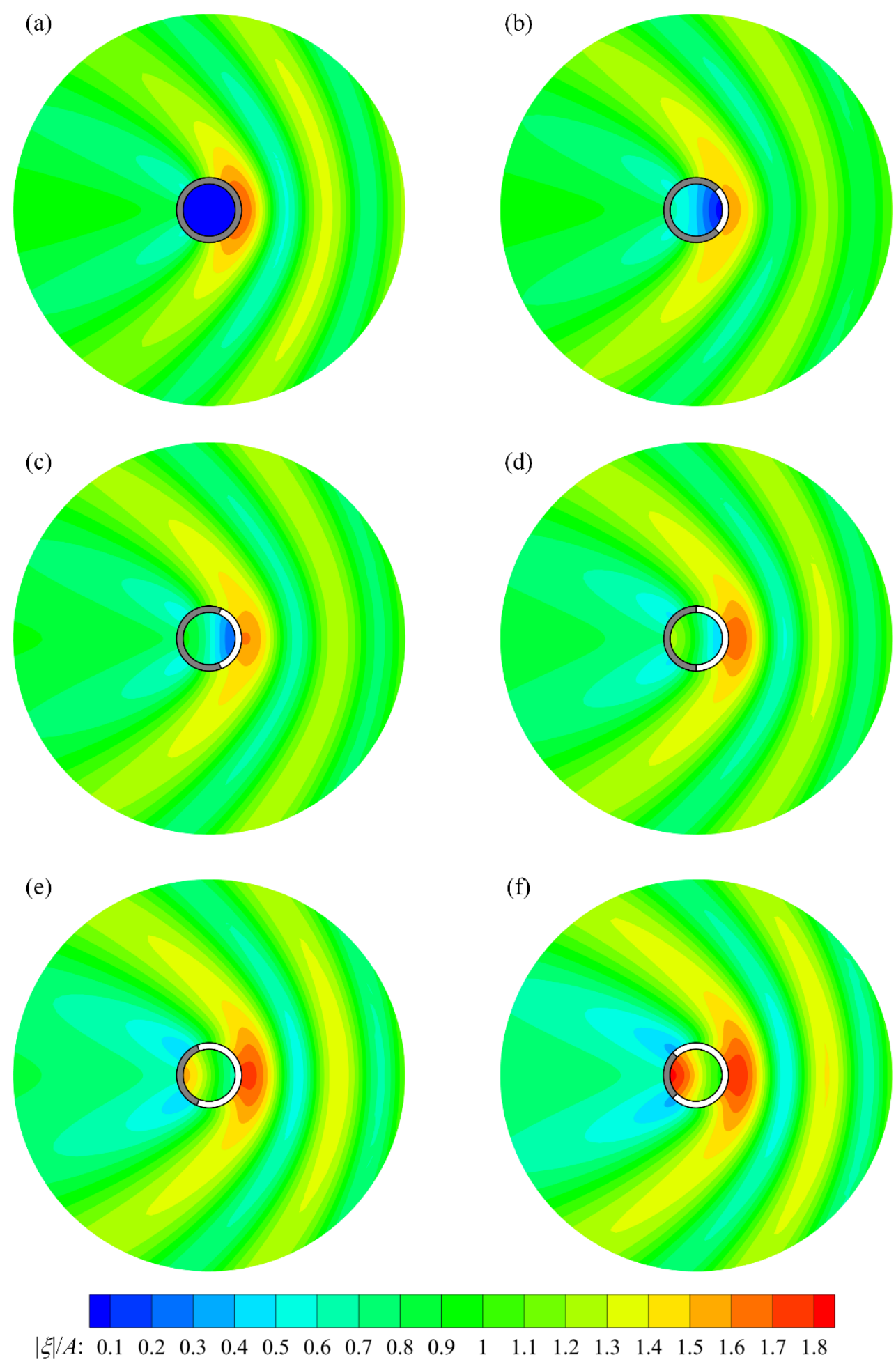

Fig. 8. Contour plot of normalized wave amplitude $(|\xi| A)$ inside and outside the tubular structure $\alpha=0.5 \pi$; (c) $\alpha=0.75 \pi$; (d) $\alpha=1.0 \pi$; (e) $\alpha=1.25 \pi$; (f) $\alpha=1.5 \pi$. In every case, $R / h=0.5,\left(R-R_{\mathrm{i}}\right) / h=0.1$, $d_{0} / h=0.3, d / h=0.2, k h=2.5$.

To carry out a quantitative analysis of the wave motion outside the chamber, the normalized wave amplitude $(\mid \xi / A)$ distributions around the tubular structure integrated OWC at $r=R$ and $r=6 R$ are plotted in Fig. 9, in which $\theta \in[0, \pi]$ is adopted to represent the whole range of $\theta$ due to the 
symmetry. As shown in Fig. 9a, $|\xi| / A$ around the OWC at $r=R$ for $\alpha=0.5 \pi$ are all weaker compared to the situation with $\alpha=0$. As $\alpha$ increases, $|\xi| / A$ for $\theta \in[0,0.2 \pi]$ increases, whereas $|\xi| / A$ for $\theta \in(0.3 \pi$, $\pi]$ decreases. For $\alpha=1.25 \pi$ and $1.5 \pi,|\xi| A$ at $\theta=0$ are 1.73 and 1.76 , respectively, which are even larger than that (1.69) for $\alpha=0$. An interesting behaviour that can be observed is that the position where the weakest wave motion occurs can be affected by $\alpha$ slightly.

Fig. $9 \mathrm{~b}$ plots $\mid \xi / A$ around the OWC at $r=6 R$. For $\theta \in[0,0.2 \pi],|\xi| / A$ at $r=6 R$ for $\alpha=0$ are significantly larger than those for the other examined cases with $\alpha \neq 0$. For $\theta \in(0.8 \pi, \pi],|\xi| / A$ at $r=6 R$ gets smaller and smaller with the increase of $\alpha$ from 0 to $1.5 \pi$. It can be concluded that the integration of the OWC with the tubular structure provides an effective attenuation influence on the wave field behind the tubular structure.

(a)

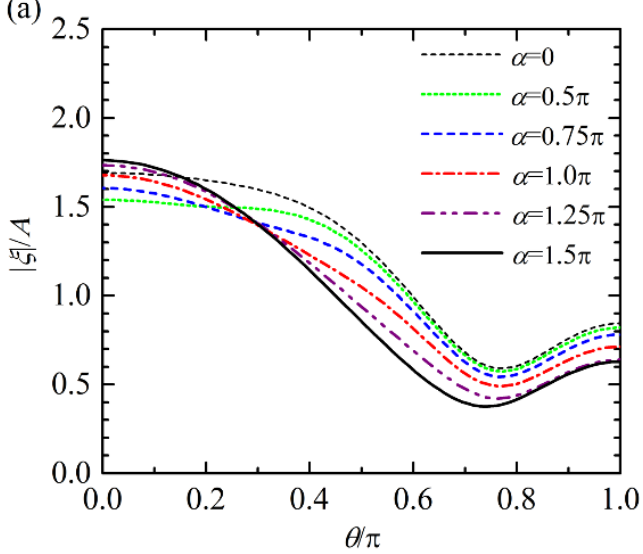

(b)

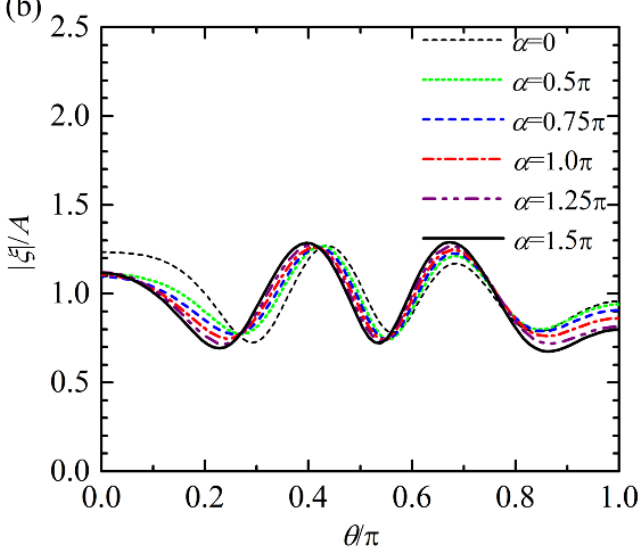

Fig. 9. Normalized wave amplitude $(|\xi| / A)$ distribution around the tubular structure integrated OWC for different opening size of the OWC chamber in terms of $\alpha$ for $R / h=0.5,\left(R-R_{\mathrm{i}}\right) / h=0.1$, $d_{0} / h=0.3, d / h=0.2, k h=2.5$. (a) $r=R$; (b) $r=6 R$.

\subsection{Opening size in terms of $d_{0}$}

Fig. 10 displays how $\left|\bar{Q}_{\mathrm{e}}\right|, \bar{c}, \bar{a}, \bar{c}_{\text {PTO }}$ and $\eta$ vary with $k h$ for five different opening size in terms of $d_{0} / h$ values $0.1,0.2,0.3,0.4$ and 0.5 , and $R / h=0.5,\left(R-R_{\mathrm{i}}\right) / h=0.1, \alpha=1.0 \pi, d / h=0.2$. Note in these five cases, submergence of the upper edge of the openings are all the same.

For a relatively small opening size with $d_{0} / h=0.1$, a very sharp peak of $\left|\bar{Q}_{\mathrm{e}}\right|$ curve can be obtained around $k h=1.0$. As $d_{0} / h$ increases, the main peaks of $\left|\bar{Q}_{\mathrm{e}}\right|$ curves are generally lower and broader with the corresponding $k h$ moving towards larger frequency. Whereas the second peaks almost remains at the same position regardless the change of $d_{0} / h$. As a comparison, as shown in Fig. 10b, although effect of $d_{0} / h$ on the bandwidth and band position of $\bar{c}$ is similar to that for $\left|\bar{Q}_{\mathrm{e}}\right|$, the height of the first peak of $\bar{c}$ roughly increases with the increase of $d_{0} / h$.

Frequency responses of $\bar{a}, \bar{c}_{\text {РTO }}$ and $\eta$ are illustrated in Figs. 10c, 10d and 10e. Since the first letter "N" of the $\bar{a}$ curve turns wider and shifts towards larger $k h$ if $d_{0} / h$ increases, the first two intersection points between the $\bar{a}$ and $-\bar{a}_{\text {PTO }}$ curves are wider apart and move together to higher frequencies. This explains why the larger value of $d_{0} / h$ corresponds to a greater bandwidth of wave power capture factor with the frequency position shifting towards larger $k h$ as shown in Fig. 10e. Additionally, the larger the $d_{0} / h$, the larger the two peak heights can be achieved at $k h<4.0$. Hence bandwidth, peak height and position of high wave power capture factor can be controlled by proper choice of the opening size in terms of $d_{0}$. 
441

442

An interesting phenomenon that deserves mention is that the effect of $d_{0} / h$ on $\left|\bar{Q}_{\mathrm{e}}\right|, \bar{c}, \bar{a}$, $\bar{c}_{\text {PTO }}$ and $\eta$ becomes weaker and weaker with the increase of $d_{0} / h$. For example, as $d_{0} / h$ increases from 0.1 to 0.2 , the largest value of $\eta$ for $k h<4.0$ changes dramatically from 2.23 at $k h=1.59$ to 2.55 at $k h=2.16$; whereas when $d_{0} / h$ increases from 0.4 to 0.5 , the largest value changes slightly from 2.74 at $k h=2.65$ to 2.77 at $k h=2.79$. This kind of results is reasonable for most wave power (95\% approximately) is concentrated at no more than a quarter wavelength below the sea water level. Therefore, in practice, it is not worth increasing the vertical extent of the OWC opening under the water at the expense of structural strength and OWC survivability.

(a)

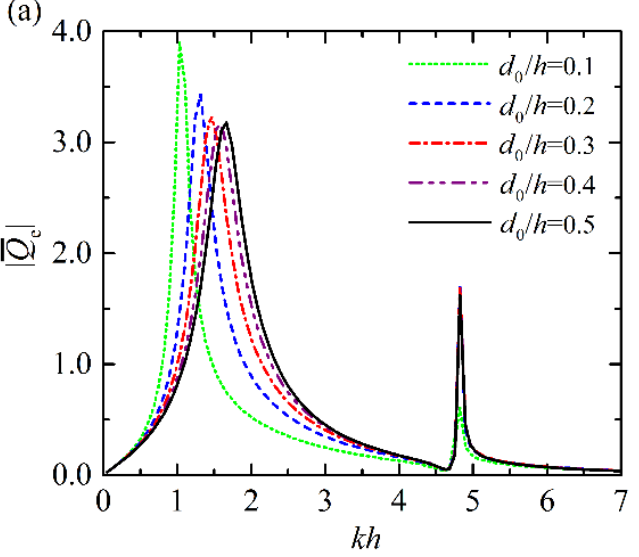

\footnotetext{
(c)
}

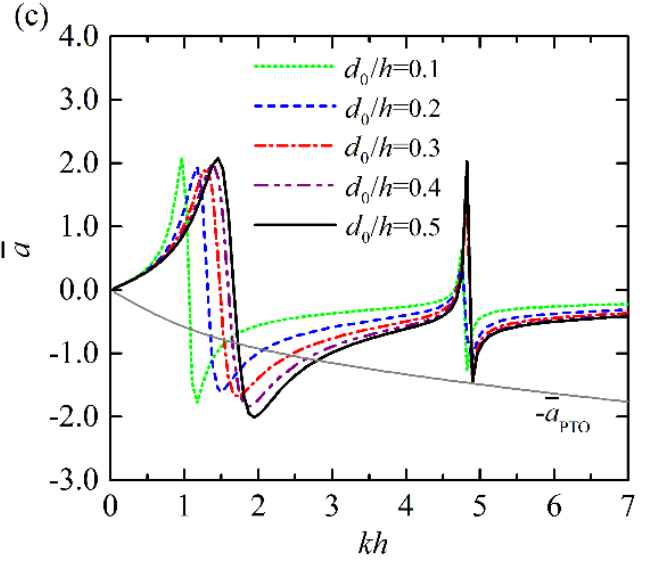

(b)

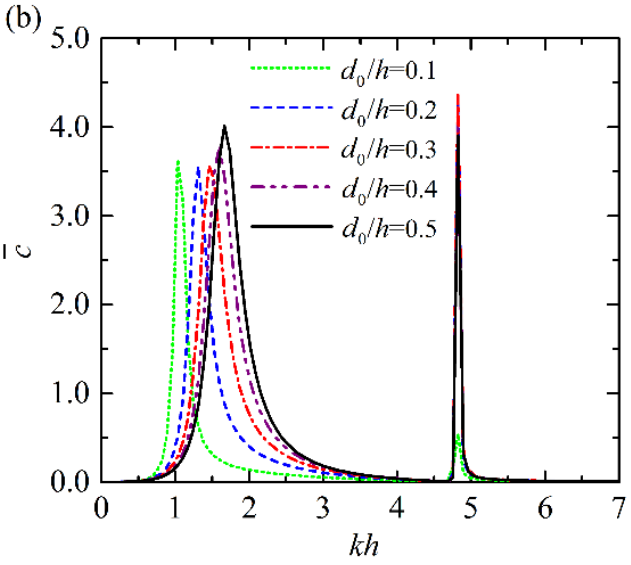

(d)

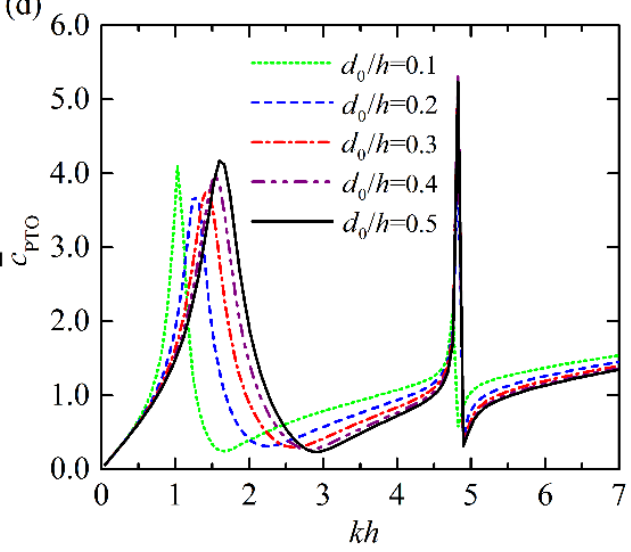

(c)

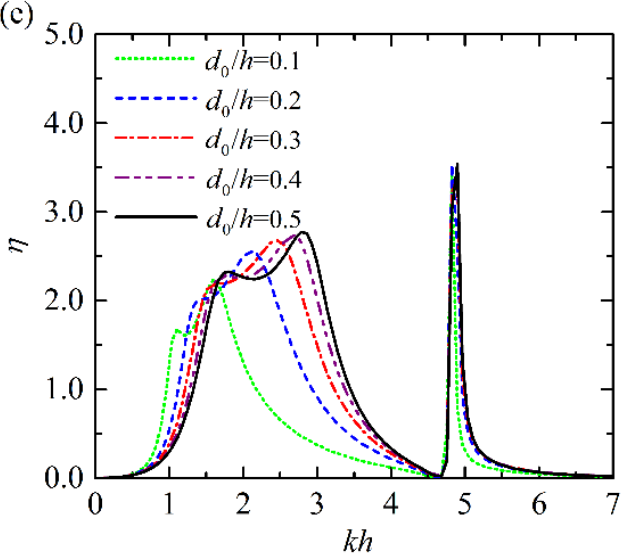

Fig. 10. Comparison for different opening size of the OWC chamber in terms of $d_{0} / h=0.1,0.2$, $0.3,0.4,0.5$. (a) $\left|\bar{Q}_{\mathrm{e}}\right|$; (b) $\bar{c}$; (c) $\bar{a}$ and $-\bar{a}_{\mathrm{PTO}}$ (gray solid line); (d) $\bar{c}_{\mathrm{PTO}}$; (e) $\eta$. In every case, 
4.5 Submerged depth of the opening, $d$

For five different submerged depths of the opening in terms of $d / h$ values $0.1,0.15,0.2,0.25$ and 0.3 , and $R / h=0.5,\left(R-R_{\mathrm{i}}\right) / h=0.1, \alpha=1.0 \pi, d_{0} / h=0.3$, Fig. 11 presents how $\left|\bar{Q}_{\mathrm{e}}\right|, \bar{c}, \bar{a}, \bar{c}_{\text {РтО }}$ and $\eta$ vary with $k h$.

As $d / h$ increases (i.e., deeper submergence of the opening), the peaks of $\left|\bar{Q}_{\mathrm{e}}\right|$ all shift towards smaller wave frequency and become narrower and sharper (see Fig. 11a). Similar changes are also observed for $\bar{c}$, as shown in Fig. 11b, and this is reasonable as the radiation loss becomes weaker for a larger values of $d / h$. Particularly, the height of the first peaks of $\left|\bar{Q}_{\mathrm{e}}\right|$ and $\bar{c}$ get larger.

Clearly, as plotted in Fig. 11c, smaller $d / h$ (i.e., shallower submergence of the opening) leads to lower and flatter curves of $\bar{a}$ within $k h<4.0$, with the sign changing point of $\bar{a}$ and also the two intersection points between the $\bar{a}$ and $-\bar{a}_{\text {PTO }}$ curves moving towards higher frequency. The optimal PTO damping $\bar{c}_{\text {PTO }}$ and the corresponding wave power capture factor $\eta$ are given in Figs. $11 \mathrm{~d}$ and 11e. Although the difference between the two resonance frequencies does not change too much with the variation of $d / h$ (see Fig.11c), the capture factor is high for a much wider primary bandwidth if $d / h$ decreases (see Fig. 11e). Similar to the primary band, the secondary band within $k h \in[4.5,7.0]$ is also broader for a smaller value of $d / h$. Therefore a smaller value of $d / h$ is welcome for the better performance in capturing wave power with a broader and smoother band.

Note in particular that in the application of the tubular structure integrated OWC, $d / h$ should be large enough to keep the opening consinuously submerged, expecially for wave conditions with large amplitude or large tidal range, or both.

(a)

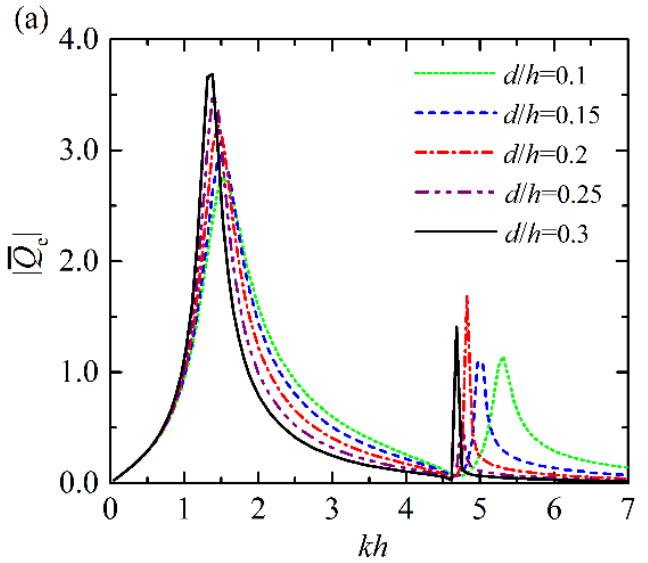

(b)

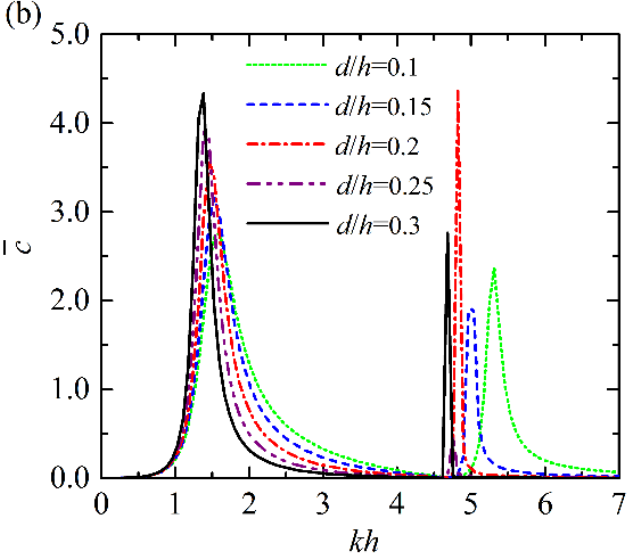




\section{(c)}

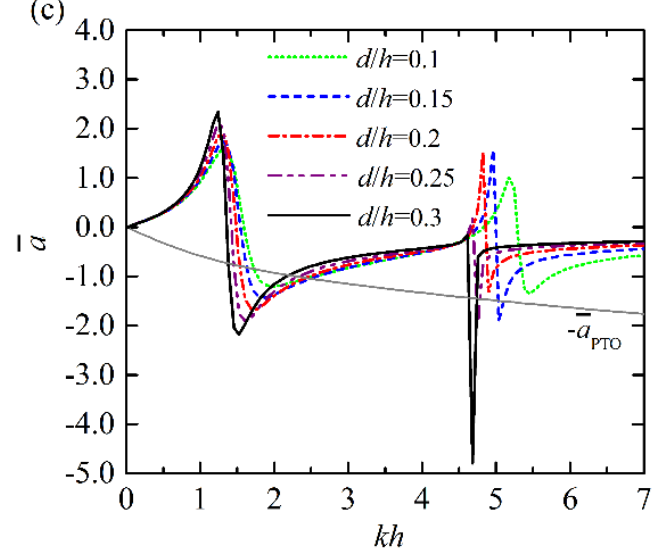

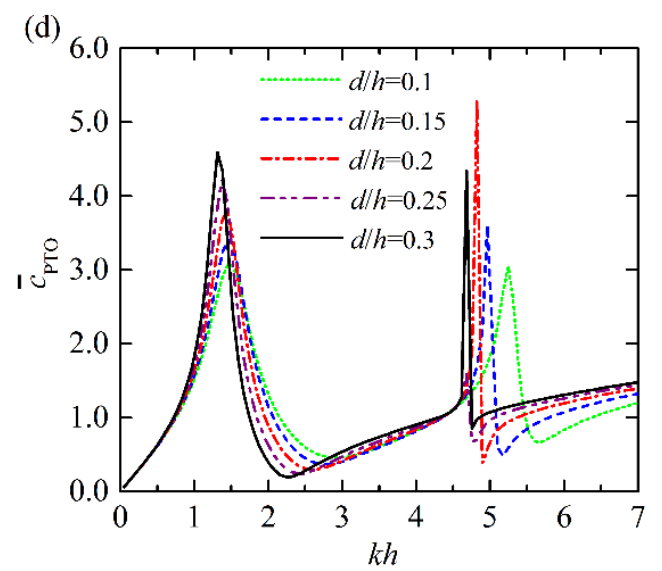

470

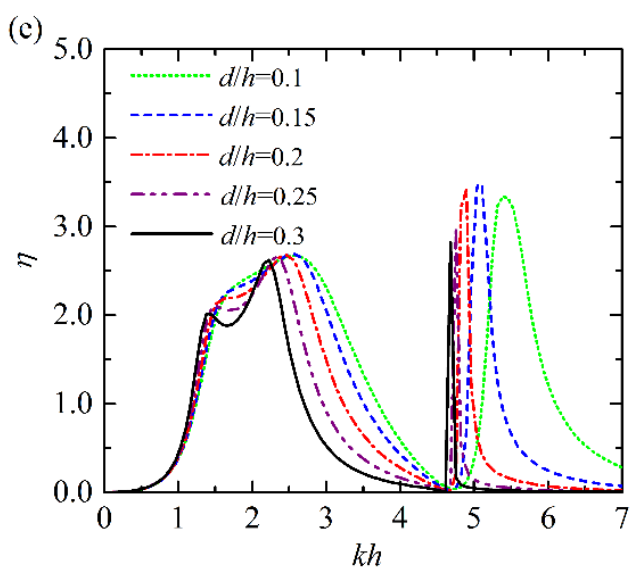

471

472

473

474

475

476

477

478

479

480

481

482

483

484

485

486

487

488

489

490

491

492

Fig. 11. Comparison for different submerged depth of the opening, $d / h=0.1,0.15,0.2,0.25,0.3$. (a) $\left|\bar{Q}_{\mathrm{e}}\right|$; (b) $\bar{c}$; (c) $\bar{a}$ and $-\bar{a}_{\text {РтО }}$ (gray solid line); (d) $\bar{c}_{\text {РтO }}$; (e) $\eta$. In every case, $R / h=0.5,(R-$ $\left.R_{\mathrm{i}}\right) / h=0.1, \alpha=1.0 \pi, d_{0} / h=0.3$.

\section{Conclusions}

In this paper, a tubular structure integrated OWC is considered in which the geometry of the chamber opening is varied, in place of an opening fully extended to the seabed. An analytical model based on potential flow theory and an eigen-function matching method accounting for the finite wall thickness of structure, is developed to address the wave diffraction and radiation problems and to further evaluate the potential power extraction of the device.

A traditional fixed hollow shaped OWC and a thin walled OWC with side opening extended to the seabed are two special cases of the varaible geometry device considered. Thus previous analyses of these two cases were used to validate the present analytical model. The validated analytical model was applied to investigate the influence of the opening size and position, and of the radius and wall thickness of the tubular structure on the power extraction of the OWC with incident waves propagating perpendicular to the opening. The following conclusions may be drawn.

With an increase in $R / h$, more resonant frequencies are found within the computed range of $k h$, i.e., $0<k h \leq 7.0$. Meanwhile, the corresponding primary band of the $\eta$ frequency response curve moves towards lower frequencies and becomes narrower.

An OWC with a thinner chamber wall thickness offers obvious benefits to wave power extraction in terms of a wider primary band of $\eta$ curves, without significantly affecting the frequency position of the band. The secondary band of $\eta$ curves is broadened at the same time, while the 
corresponding $k h$ turns smaller. In practice, $\left(R-R_{\mathrm{i}}\right) / h$ is not allowed to be too small for the sake of structural strength and OWC survivability.

As $\alpha$ increases from $0.5 \pi$ to $1.5 \pi$, the primary band of the $\eta$ curve shifts towards higher frequencies with enhanced bandwidth, whereas the peak value of $\eta$ is first weakened and then strengthened.

In particular, the study of wave motion around the OWC for $k h=2.5$ reveals that the maximum and minimum wave amplitudes predicted inside the chamber occur at the innermost of the OWC chamber and at the opening, respectively. The larger the value of $\alpha$, the stronger the wave motion inside the chamber. The largest wave motion outside the OWC occurs in front of the opening regardless of its size. It is interesting to note that integration of the OWC within the tubular structure provides an effective attenuation influence on the wave field behind the structure.

With increasing $d_{0} / h$, a broader and higher band of wave power capture factor with the frequency position shifting towards larger $k h$ is achieved. Performance of the OWC becomes more and more insensitive with the increase of $d_{0} / h$, hence it is not essential to open the side wall of the OWC too large vertically under the water - which might cause problems of structural strength and OWC survivability.

A smaller value of $d / h$ is advantageous in terms of wave power capture factor, leading to a broader and smoother band, whilst observing that $d / h$ should also be kept deep enough to ensure the opening remains continuously submerged.

The potential flow based analytical model will not capture viscous effects, hence it is not suitable for extreme wave structure interactions and may over predict the performance of the WEC under operational conditions. This paper deals with an isolated tubular structure integrated OWC. For an array of these OWCs with small spacing perhaps in the case of theintegration of OWCs into a pile breakwater, then the hydrodynamic interactions between them may play a significant role in their overall power extraction performance. The present analytical model can be extended to study the performance of multiple tubular structures with integrated OWC, which will be reported elsewhere.

\section{Acknowledgements}

The research was supported by Intelligent Community Energy (ICE), INTERREG V FCE, European Commission (Contract No. 5025). The second author gratefully acknowledges the financial support from China Scholarship Council (Grant No. 201806060137). 
Appendix A. Derivation of equations for solving the scattering and radiation problems

After inserting Eqs. (11) and (15) into Eq.(20), multiplying both sides by $Z_{\zeta}(z) \mathrm{e}^{-\mathrm{i} t \theta}$ and integrating for $z \in[-h, 0]$ and $\theta \in[0,2 \pi]$, for any pair of integer $(\tau, \zeta)$, it can be obtained that

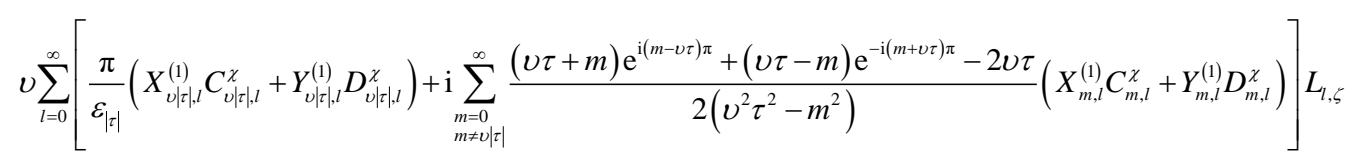

$-2 \pi h A_{t, \zeta}^{\chi}=0$

where

$$
X_{\tau, \zeta}^{(1)}=\left\{\begin{array}{l}
\frac{\tau}{v R}\left(\frac{R_{\mathrm{i}}}{R}\right)^{\frac{\tau}{v}-1}, \quad \zeta=0 \\
\frac{\beta_{\zeta} I_{\frac{\tau}{v}}^{\prime}\left(\beta_{\zeta} R_{\mathrm{i}}\right)}{I_{\frac{\tau}{v}}\left(\beta_{\zeta} R\right)}, \quad \zeta \neq 0
\end{array} \quad ; \quad Y_{\tau, \zeta}^{(1)}=\left\{\begin{array}{l}
\frac{1}{R_{\mathrm{i}}}, \quad \zeta=0, \tau=0 \\
-\frac{\tau}{v R}\left(\frac{R}{R_{\mathrm{i}}}\right)^{\frac{\tau}{v}+1}, \quad \zeta=0, \tau \neq 0, \\
\frac{\beta_{\zeta} K_{\frac{\tau}{v}}^{\prime}\left(\beta_{\zeta} R_{\mathrm{i}}\right)}{K_{\frac{\tau}{v}}\left(\beta_{\zeta} R\right)}, \quad \zeta \neq 0
\end{array}\right.\right.
$$

$$
\begin{aligned}
& L_{l, \zeta}=\int_{-h_{0}}^{-d} \cos \left[\beta_{l}\left(z+h_{0}\right)\right] Z_{\zeta}(z) \mathrm{d} z \\
& = \begin{cases}\frac{k_{0} Z_{0}(0)\left(h_{0}-d\right)^{2}\left\{(-1)^{l} \sinh \left[k_{0}(h-d)\right]-\sinh \left[k_{0}\left(h-h_{0}\right)\right]\right\}}{\left[\left(h_{0}-d\right)^{2} k_{0}^{2}+l^{2} \pi^{2}\right] \cosh \left(k_{0} h\right)}, & \zeta=0 . \\
\frac{k_{\zeta} Z_{\zeta}(0)\left(h_{0}-d\right)^{2}\left\{(-1)^{l} \sin \left[k_{\zeta}(h-d)\right]-\sin \left[k_{\zeta}\left(h-h_{0}\right)\right]\right\}}{\left[\left(h_{0}-d\right)^{2} k_{\zeta}^{2}-l^{2} \pi^{2}\right] \cos \left(k_{\zeta} h\right)}, & \zeta \neq 0\end{cases}
\end{aligned}
$$

After inserting Eqs. (15) and (18) into Eq.(21), multiplying both sides by $Z_{\zeta}(z) \mathrm{e}^{-\mathrm{i} \tau \theta}$ and integrating for $z \in[-h, 0]$ and $\theta \in[0,2 \pi]$, for any pair of integer $(\tau, \zeta)$, we have

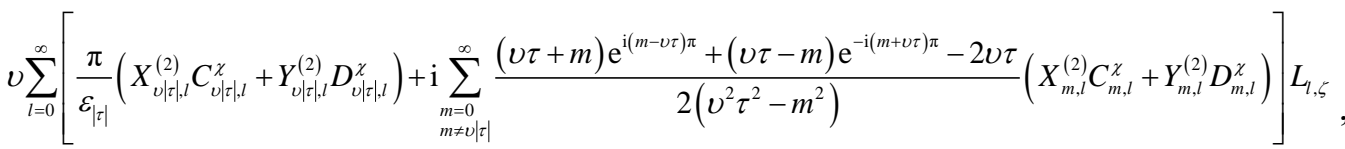

$$
\begin{aligned}
& -2 \pi h Z_{\tau, \zeta}^{(2)} E_{\tau, \zeta}^{\chi}=f_{2}^{\chi}
\end{aligned}
$$

in which

$$
\begin{gathered}
f_{2}^{\chi}= \begin{cases}-\frac{2 \pi \delta_{\zeta, 0} \mathrm{i} g A k_{0} h}{\omega Z_{0}(0)} \mathrm{i}^{\tau} J_{\tau}^{\prime}\left(k_{0} R\right) \mathrm{e}^{-\mathrm{i} \tau \beta}, & \chi=\mathrm{S}, \\
0, & \chi=\mathrm{R}\end{cases} \\
X_{\tau, \zeta}^{(2)}=\left\{\begin{array}{ll}
\frac{\tau}{v R}, & \zeta=0 \\
\frac{\beta_{\zeta} I_{\frac{\tau}{v}}^{\prime}\left(\beta_{\zeta} R\right)}{I_{\frac{\tau}{v}}\left(\beta_{\zeta} R\right)}, & \zeta \neq 0
\end{array} \quad ; \quad Y_{\tau, \zeta}^{(2)}= \begin{cases}\frac{1}{R}, & \zeta=0, \tau=0 \\
-\frac{\tau}{v R}, & \zeta=0, \tau \neq 0, \\
\frac{\beta_{\zeta} K_{\frac{\tau}{v}}^{\prime}\left(\beta_{\zeta} R\right)}{K_{\frac{\tau}{v}}\left(\beta_{\zeta} R\right)}, & \zeta \neq 0\end{cases} \right.
\end{gathered}
$$




$$
Z_{\tau, \zeta}^{(2)}=\left\{\begin{array}{ll}
\frac{k_{0} H_{\tau}^{\prime}\left(k_{0} R\right)}{H_{\tau}\left(k_{0} R\right)}, & \zeta=0 \\
\frac{k_{\zeta} K_{\tau}^{\prime}\left(k_{\zeta} R\right)}{K_{\tau}\left(k_{\zeta} R\right)}, & \zeta=1,2,3 \cdots
\end{array} .\right.
$$

After inserting Eqs. (11) and (15) into Eq.(22), multiplying both sides by $\cos \left[\beta_{\zeta}\left(z+h_{0}\right)\right] \cos (\tau \theta / v)$ and integrating for $z \in\left[-h_{0},-d\right]$ and $\theta \in[0, v \pi]$, for any pair of integer $(\tau, \zeta)$, it can be obtained that

$\sum_{l=0}^{\infty}\left(\frac{\pi}{2}\left(\frac{\frac{\tilde{I}_{\tau}}{v}\left(k_{l} R_{\mathrm{i}}\right)}{k_{l} \tilde{I}_{\frac{\tau}{v}}^{\prime}\left(k_{l} R_{\mathrm{i}}\right)} A_{\frac{\tau}{v}, l}^{\chi}+\frac{\tilde{I}_{-\frac{\tau}{v}}\left(k_{l} R_{\mathrm{i}}\right)}{k_{l} \tilde{I}_{-\frac{\tau}{v}}^{\prime}\left(k_{l} R_{\mathrm{i}}\right)} A_{-\frac{\tau}{v}, l}^{\chi}\right)-\mathrm{i} \sum_{\substack{m=-\infty \\ m v \neq \pm \tau}}^{\infty} \frac{(m v-\tau) \mathrm{e}^{\mathrm{i}(m v+\tau) \pi}+(m v+\tau) \mathrm{e}^{\mathrm{i}(m v-\tau) \pi}-2 m v}{2\left(m^{2} v^{2}-\tau^{2}\right)} \frac{\tilde{I}_{m}\left(k_{l} R_{\mathrm{i}}\right)}{k_{l} \tilde{I}_{m}^{\prime}\left(k_{l} R_{\mathrm{i}}\right)} A_{m, l}^{\chi}\right) L_{\zeta, l}$ $=\frac{\pi\left(h_{0}-d\right)}{\varepsilon_{\tau} \varepsilon_{\zeta}}\left(X_{\tau, \zeta}^{(3)} C_{\tau, \zeta}^{\chi}+Y_{\tau, \zeta}^{(3)} D_{\tau, \zeta}^{\chi}\right)+f_{3}^{\chi}$

where

$$
\begin{aligned}
& f_{3}^{\chi}= \begin{cases}0, & \chi=\mathrm{S} \\
\frac{\delta_{\tau, 0} \delta_{\zeta, 0} \mathrm{i} \pi\left(h_{0}-d\right)}{\rho \omega}, & \chi=\mathrm{R},\end{cases} \\
& X_{m, l}^{(3)}=\left\{\begin{array}{l}
\left(\frac{R_{\mathrm{i}}}{R}\right)^{\frac{m}{v}}, \quad l=0 \\
\frac{I_{\frac{m}{v}}\left(\beta_{l} R_{\mathrm{i}}\right)}{I_{\frac{m}{v}}\left(\beta_{l} R\right)}, \quad l \neq 0
\end{array} ; \quad Y_{m, l}^{(3)}=\left\{\begin{array}{l}
1+\ln \left(\frac{R_{\mathrm{i}}}{R}\right), \quad l=0, m=0 \\
\left(\frac{R}{R_{\mathrm{i}}}\right)^{\frac{m}{v}}, \quad l=0, m \neq 0 \\
\frac{K_{\frac{m}{v}}\left(\beta_{l} R_{\mathrm{i}}\right)}{K_{\frac{m}{v}}\left(\beta_{l} R\right)}, \quad l \neq 0
\end{array} .\right.\right.
\end{aligned}
$$

After inserting Eqs. (15) and (18) into Eq.(23), multiplying both sides by $\cos \left[\beta_{\zeta}\left(z+h_{0}\right)\right] \cos (\tau \theta / v)$ and integrating for $z \in\left[-h_{0},-d\right]$ and $\theta \in[0, v \pi]$, for any pair of integer $(\tau, \zeta)$, we have

$$
\begin{aligned}
& \sum_{l=0}^{\infty}\left(\frac{\pi}{2}\left(E_{\frac{\tau}{v}, l}^{\chi}+E_{-\frac{\tau}{v}, l}^{\chi}\right)-\mathrm{i} \sum_{\substack{m=-\infty \\
|m| v \neq \tau}}^{\infty} \frac{(m v-\tau) \mathrm{e}^{\mathrm{i}(m v+\tau) \pi}+(m v+\tau) \mathrm{e}^{\mathrm{i}(m v-\tau) \pi}-2 m v}{2\left(m^{2} v^{2}-\tau^{2}\right)} E_{m, l}^{\chi}\right) L_{\zeta, l}, \\
& =\frac{\pi\left(h_{0}-d\right)}{\varepsilon_{\tau} \varepsilon_{\zeta}}\left(C_{\tau, \zeta}^{\chi}+D_{\tau, \zeta}^{\chi}\right)+f_{4}^{\chi}
\end{aligned}
$$

where

$$
f_{4}^{\chi}=\left\{\begin{array}{lc}
\frac{\mathrm{i} g A L_{\zeta, 0}}{\omega Z_{0}(0)}\left(\pi \mathrm{i}^{\frac{\tau}{\nu}} J_{\frac{\tau}{v}}\left(k_{0} R\right) \cos \left(\frac{\tau}{v} \beta\right)-\mathrm{i} \sum_{\substack{m=-\infty \\
m v \neq \tau}}^{\infty} \frac{(m v-\tau) \mathrm{e}^{\mathrm{i}(m v+\tau) \pi}+(m v+\tau) \mathrm{e}^{\mathrm{i}(m v-\tau) \pi}-2 m v}{2\left(m^{2} v^{2}-\tau^{2}\right)} \mathrm{i}^{m} \mathrm{e}^{-\mathrm{i} m \beta} J_{m}\left(k_{0} R\right)\right), \chi=\mathrm{S} \\
0, \quad \chi(A) \mathrm{R}
\end{array}\right.
$$

Eqs. (A.1), (A.4), (A.8) and (A.11) form a linear algebraic system, which can be used to solve $A_{m, l}^{\chi}, C_{m, l}^{\chi}, D_{m, l}^{\chi}$ and $E_{m, l}^{\chi}$ numerically after truncation. In the present model, the infinite terms of $\mathrm{e}^{-\mathrm{i} m \theta} / \cos (m \theta / v)$, and $Z_{l}(z) / \cos \left[\beta_{l}\left(z+h_{0}\right)\right]$ are truncated at $m=M$ and $l=L$, respectively. Accurate results can be obtained by choosing $M=12, L=20$. 


\section{References}

Astariz, S., Iglesias, G., 2015. The economics of wave energy: A review. Renewable \& Sustainable Energy Reviews 45, 397-408.

Bosma, B., Brekken, T., Lomonaco, P., McKee, A., Paasch, B., Batten, B., 2017. Physical model testing and system identification of a cylindrical OWC device, Proceedings of the 12th European Wave and Tidal Energy Conference, Cork, Ireland, pp. 1-10.

Clément, A., McCullen, P., Falcão, A., Fiorentino, A., Gardner, F., Hammarlund, K., Lemonis, G., Lewis, T., Nielsen, K., Petroncini, S., Pontes, M.T., Schild, P., Sjöström, B.O., Sørensen, H.C., Thorpe, T., 2002. Wave energy in Europe: current status and perspectives. Renewable \& Sustainable Energy Reviews 6 (5), $405-$ 431.

Contestabile, P., luppa, C., Lauro, E.D., Cavallaro, L., Andersen, T.L., Vicinanza, D., 2017. Wave loadings acting on innovative rubble mound breakwater for overtopping wave energy conversion. Coastal Engineering 122, 60-74.

Deng, Z.Z., Huang, Z.H., Law, A.W.K., 2013. Wave power extraction by an axisymmetric oscillating-watercolumn converter supported by a coaxial tube-sector-shaped structure. Applied Ocean Research 42, 114-123.

Deng, Z.Z., Huang, Z.H., Law, A.W.K., 2014. Wave power extraction from a bottom-mounted oscillating water column converter with a V-shaped channel. Proceedings of the Royal Society a-Mathematical Physical and Engineering Sciences 470 (2167).

Drew, B., Plummer, A.R., Sahinkaya, M.N., 2009. A review of wave energy converter technology. Proceedings of the Institution of Mechanical Engineers Part a-Journal of Power and Energy 223 (A8), 887-902.

Elhanafi, A., Fleming, A., Macfarlane, G., Leong, Z., 2017. Underwater geometrical impact on the hydrodynamic performance of an offshore oscillating water column-wave energy converter. Renewable Energy 105, 209-231.

Evans, D.V., Porter, R., 1995. Hydrodynamic characteristics of an oscillating water column device. Applied Ocean Research 17 (3), 155-164.

Falcão, A.F.d.O., Henriques, J.C.C., 2016. Oscillating-water-column wave energy converters and air turbines: A review. Renewable Energy 85, 1391-1424.

Falnes, J., 2002. Ocean Waves and Oscillating Systems: Linear Interaction Including Wave-energy Extraction. Cambridge University Press, Cambridge, UK.

Guo, B.Y., Patton, R., Jin, S.Y., Gilbert, J., Parsons, D., 2018. Nonlinear modeling and verification of a heaving point absorber for wave energy conversion. leee Transactions on Sustainable Energy 9 (1), 453 461.

He, F., Huang, Z., Law, A.W.K., 2013. An experimental study of a floating breakwater with asymmetric pneumatic chambers for wave energy extraction. Applied Energy 106, 222-231.

He, F., Huang, Z.H., 2014. Hydrodynamic performance of pile-supported OWC-type structures as breakwaters: An experimental study. Ocean Engineering 88, 618-626.

Henriques, J.C.C., Cândido, J.J., Pontes, M.T., Falcão, A.F.O., 2013. Wave energy resource assessment for a breakwater-integrated oscillating water column plant at Porto, Portugal. Energy 63, 52-60.

Lovas, S., Mei, C.C., Liu, Y.M., 2010. Oscillating water column at a coastal corner for wave power extraction. Applied Ocean Research 32 (3), 267-283.

López, I., Iglesias, G., 2014. Efficiency of OWC wave energy converters: A virtual laboratory. Applied 
Ocean Research 44, 63-70.

López, I., Pereiras, B., Castro, F., Iglesias, G., 2016. Holistic performance analysis and turbine-induced damping for an OWC wave energy converter. Renewable Energy 85, 1155-1163.

Martins-rivas, H., Mei, C.C., 2009a. Wave power extraction from an oscillating water column along a straight coast. Ocean Engineering 36 (6-7), 426-433.

Martins-rivas, H., Mei, C.C., 2009b. Wave power extraction from an oscillating water column at the tip of a breakwater. Journal of Fluid Mechanics 626, 395-414.

Morris-Thomas, M.T., Irvin, R.J., Thiagarajan, K.P., 2007. An investigation into the hydrodynamic efficiency of an oscillating water column. Journal of Offshore Mechanics and Arctic Engineering 129 (4), 273-278.

Mustapa, M.A., Yaakob, O.B., Ahmed, Y.M., Rheem, C.K., Koh, K.K., Adnan, F.A., 2017. Wave energy device and breakwater integration: A review. Renewable \& Sustainable Energy Reviews 77, 43-58.

Nader, J.R., 2013. Interaction of ocean waves with oscillating water column wave energy convertors, School of Mathematics and Applied Sciences. University of Wollongong, Wollongong.

Ning, D.Z., Zhao, X.L., Chen, L.F., Zhao, M., 2018. Hydrodynamic performance of an array of wave energy converters integrated with a pontoon-type breakwater. Energies 11 (3), 685.

Perez-Collazo, C., Greaves, D., Iglesias, G., 2018a. Hydrodynamic response of the WEC sub-system of a novel hybrid wind-wave energy converter. Energy Conversion and Management 171, 307-325.

Perez-Collazo, C., Greaves, D., Iglesias, G., 2018b. A novel hybrid wind-wave energy converter for jacketframe substructures. Energies 11 (3), 637.

Rezanejad, K., Bhattacharjee, J., Guedes Soares, C., 2013. Stepped sea bottom effects on the efficiency of nearshore oscilating water column device. Ocean Engineering 70, 25-38.

Rezanejad, K., Bhattacharjee, J., Guedes Soares, C., 2015. Analytical and numerical study of dualchamber oscillating water columns on stepped bottom. Renewable Energy 75, 272-282.

Rezanejad, K., Bhattacharjee, J., Guedes Soares, C., 2016. Analytical and numerical study of nearshore multiple oscillating water columns. Journal of Offshore Mechanics and Arctic Engineering 138 (021901). Sarmento, A.J.N.A., Falcão, A.F.d.O., 1985. Wave Generation by an Oscillating Surface-Pressure and Its Application in Wave-Energy Extraction. Journal of Fluid Mechanics 150 (Jan), 467-485.

Sheng, W., Alcorn, R., Lewis, A., 2014a. Assessment of primary energy conversions of oscillating water columns. I. Hydrodynamic analysis. Journal of Renewable and Sustainable Energy 6 (5), 053113.

Sheng, W., Alcorn, R., Lewis, A., 2014b. Assessment of primary energy conversions of oscillating water columns. II. Power take-off and validations. Journal of Renewable and Sustainable Energy 6 (5), 053114. Viviano, A., Naty, S., Foti, E., Bruce, T., Allsop, W., Vicinanza, D., 2016. Large-scale experiments on the behavior of a generalized Oscillating Water Column under random waves. Renewable Energy 99, 875887.

Xu, C., Huang, Z., 2018. A dual-functional wave-power plant for wave-energy extraction and shore protection: A wave-flume study. Applied Energy 229, 963-976.

Zhao, X.L., Ning, D.Z., Liang, D.F., 2019. Experimental investigation on hydrodynamic performance of a breakwater-integrated WEC system. Ocean Engineering 171, 25-32.

Zheng, S., Antonini, A., Zhang, Y., Greaves, D., Miles, J., Iglesias, G., 2019a. Wave power extraction from multiple oscillating water columns along a straight coast. Journal of Fluid Mechanics 878, 445-480.

Zheng, S., Zhang, Y., 2015. Wave diffraction from a truncated cylinder in front of a vertical wall. Ocean Engineering 104, 329-343.

Zheng, S., Zhang, Y., 2016. Wave radiation from a truncated cylinder in front of a vertical wall. Ocean 
641 Zheng, S., Zhang, Y., 2017. Analysis for wave power capture capacity of two interconnected floats in 642 regular waves. Journal of Fluids and Structures 75, 158-173.

643 Zheng, S., Zhang, Y., 2018. Theoretical modelling of a new hybrid wave energy converter in regular waves.

644 Renewable Energy 128A, 125-141.

645 Zheng, S., Zhang, Y., Iglesias, G., 2018. Wave-structure interaction in hybrid wave farms. Journal of Fluids 646 and Structures 83, 386-412.

647 Zheng, S., Zhang, Y., Iglesias, G., 2019b. Coast/breakwater-integrated OWC: A theoretical model. Marine 648 Structures 66, 121-135.

649 Zheng, S.M., Zhang, Y.H., Zhang, Y.L., Sheng, W.A., 2015. Numerical study on the dynamics of a two-raft 650 wave energy conversion device. Journal of Fluids and Structures 58, 271-290.

651 